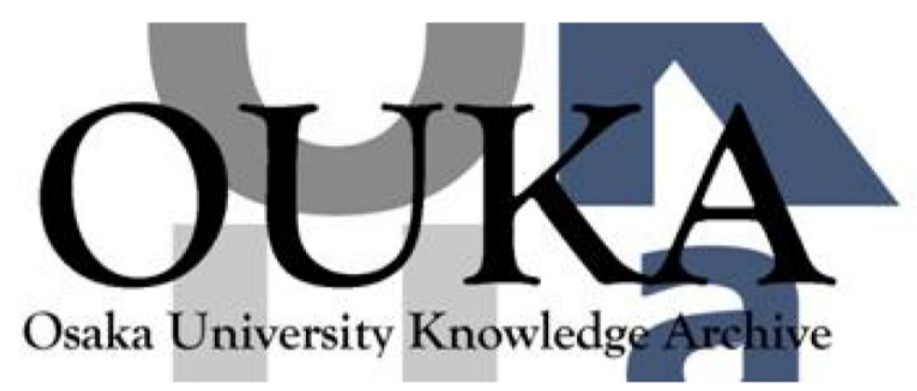

\begin{tabular}{|c|c|}
\hline Title & $\begin{array}{l}\text { Ion-temperature-gradient-driven transport in a } \\
\text { density modification experiment on the tokamak } \\
\text { fusion test reactor }\end{array}$ \\
\hline Author (s) & Horton, W. ; Lindberg, D.; Kim, J. Y. et al. \\
\hline Citation & Physics of Fluids B. 4(4) p.953-p. 966 \\
\hline Issue Date & $1992-04$ \\
\hline oaire:version & VoR \\
\hline URL & https://hdl. handle. net/11094/78520 \\
\hline rights & $\begin{array}{l}\text { This article may be downloaded for personal use } \\
\text { only. Any other use requires prior permission } \\
\text { of the author and AIP Publishing. This article } \\
\text { appeared in Physics of Fluids B: Plasma Physics } \\
4,953 \text { (1992) and may be found at } \\
\text { https://doi.org/10.1063/1.860112. }\end{array}$ \\
\hline Note & \\
\hline
\end{tabular}

Osaka University Knowledge Archive : OUKA

https://ir. Library. osaka-u. ac. jp/

Osaka University 


\section{Ion-temperature-gradient-driven transport in a density modification experiment on the Tokamak Fusion Test Reactor}

Cite as: Physics of Fluids B: Plasma Physics 4, 953 (1992); https://doi.org/10.1063/1.860112 Submitted: 30 September 1991 . Accepted: 15 January 1991 . Published Online: 04 June 1998

W. Horton, D. Lindberg, J. Y. Kim, J. Q. Dong, G. W. Hammett, S. D. Scott, M. C. Zarnstorff, and S. Hamaguchi

\section{ARTICLES YOU MAY BE INTERESTED IN}

Electron temperature gradient driven turbulence

Physics of Plasmas 7, 1904 (2000); https://doi.org/10.1063/1.874014

Comparisons and physics basis of tokamak transport models and turbulence simulations Physics of Plasmas 7, 969 (2000); https://doi.org/10.1063/1.873896

Ion temperature-gradient-driven modes and anomalous ion transport in tokamaks Physics of Fluids B: Plasma Physics 1, 1018 (1989); https://doi.org/10.1063/1.859023 


\title{
lon-temperature-gradient-driven transport in a density modification experiment on the Tokamak Fusion Test Reactor
}

\author{
W. Horton, D. Lindberg, J. Y. Kim, and J. Q. Dong \\ Institute for Fusion Studies, The University of Texas at Austin, Austin, Texas 78712 \\ G. W. Hammett, S. D. Scott, and M. C. Zarnstorff \\ Princeton Plasma Physics Laboratory, P.O. Box 451, Princeton, New Jersey 08544 \\ S. Hamaguchi \\ IBM Research Division, Thomas J. Watson Research Center, Yorktown Heights, New York 10598
}

(Received 30 September 1991; accepted 15 January 1992)

\begin{abstract}
Tokamak Fusion Test Reactor (TFTR) profiles from a supershot density-modification experiment [Zarnstorff et al., Plasma Physics and Controlled Nuclear Fusion Research, 1990, Proceedings of the 12th International Conference, Washington (IAEA, Vienna, 1991), Vol. I, p. 109] are analyzed for their local and ballooning stability to toroidal $\eta_{i}$ modes in order to understand the initially puzzling results showing no increase in $\chi_{i}$ when a pellet is used to produce an abrupt and large increase in the $\eta_{i}$ parameter. The local stability analysis assumes that $k_{\|}=1 / q R$ and ignores the effects of shear, but makes no assumption on the magnitude of $k_{\|} v_{t i} / \omega$. The ballooning stability analysis determines a self-consistent linear spectrum of $k_{\|}$'s including the effect of shear and toroidicity, but it expands in $k_{\|} v_{t i} / \omega \leqslant 1$, which is a marginal assumption for this experiment. Nevertheless, the two approaches agree well and show that the mixing length estimate of the transport rate does not change appreciably during the density modification and has a value close to or less than the observed $\chi_{i}$, in contrast to most previous theories, which predicted $\chi_{i}$ 's that were over an order-of-magnitude too large. However, still to be explained is the observed increase of $\chi_{i}(r)$ with minor radius by adding the effects of (i) the finite-beta drift wave-magnetohydrodynamic (MHD) mode coupling, (ii) the slablike mode, or (iii) the trapped-electron response. The experimental tracking $0.2<\chi_{e} / \chi_{i}<0.7$ suggests that both grad $T_{i}$ and trapped-electron driving mechanisms are operating.
\end{abstract}

\section{INTRODUCTION}

Transport studies in the large tokamak confinement devices show that the ion and electron thermal transport rates are well above the collisional neoclassical transport rates. When the thermal losses are expressed in terms of thermal diffusivities $\chi_{i}$ and $\chi_{e}$, the lost rates are characterized as having comparable diffusivities $\chi_{i} \sim \chi_{e}$ with the order-ofmagnitude of $\chi$ consistent with the $\mathbf{E} \times \mathbf{B}$ transport diffusion expected from small-scale drift wave turbulence. Drift wave stability theory predicts that typical tokamak discharges are unstable to drift waves driven by both the ion and the electron temperature and density gradients. An important stability parameter controlling the onset and the strength of the turbulence is the ratio of the density gradient scale length $L_{n}$ to the temperature gradient scale length $L_{T}$ called the eta parameter $\eta=L_{n} / L_{T}$.

Early pellet fueling experiments in the Alcator-C tokamak' showed the onset of improved confinement with the steepening of the density profile which is readily interpreted in terms of the ion temperature gradient drift wave turbulence due to the simultaneous sharp decrease in the $\eta_{i}$ stability parameter. Similarly, improved confinement regimes in numerous other machines have been interpreted in terms of steepening the density gradient so as to lower the $\eta_{i}$ and $\eta_{e}$ stability parameters. In such an example, the ASDEX team ${ }^{2}$ used density profile control to extend the unsaturated Alca- tor energy confinement scaling $\tau_{E} \propto \bar{n}_{e}$ by a factor of 2 above the original saturation limit.

These various transport results show the need for a detailed study of the ion thermal transport in terms of drift wave turbulence theory. A series of transport studies on the Tokamak Fusion Test Reactor (TFTR) were undertaken by Scott et al.$^{3,4}$ and Zarnstorff et al. ${ }^{5,6}$ to test the hypothesis that the ion transport is due to the ion-temperature-gradientdriven drift wave turbulence.

Earlier comparisons of TFTR experimental results with the existing ion temperature gradient (ITG) theories yielded mixed results. A set of measurements ${ }^{3}$ in the hot-ion and supershot regimes showed that theories gave $\chi_{i}$ 's that were $10-100$ times too big in the plasma core $(r<a / 3)$. However, these theories were derived in the $\eta_{i} \gg \eta_{\text {crit }}$ limit and did not contain a smooth transition to zero transport as $\eta_{i}$ dropped below $\eta_{\text {crit }}$ where the ITG mode becomes stable. It was observed that these plasmas were actually close to marginal stability (see Fig. 2 of Ref. 3 ), which would explain the differences between the measured and theoretical $\chi_{i}$. In fact, the correlation of the measured $\eta_{i}$ with the theoretical $\eta_{\text {crit }}$ (see Fig. 4 of Ref. 3) suggested that the plasma was forced to stay near marginal stability by the strong ITG transport, which would result if $\eta_{i} \gg \eta_{\text {crit }}$. A later set of experiments, ${ }^{4}$ which included L-mode plasmas found that some plasmas were able to have $\eta_{i} \gg \eta_{\text {crit }}$, but these tended to be colder plasmas for which the theoretical $\chi_{i}$ was perhaps not strong 
enough to enforce marginal stability.

These findings lead to the experiments by Zarnstorff et al., 5,6 where the density profile of a hot supershot plasma was modified by a deuterium pellet or by helium gas injection to flatten the density profile and force $\eta_{i} \gg \eta_{\text {crit }}$. It was expected that a very large $\chi_{i}$ would then be observed in the experiment, but, in fact, $\chi_{i}$ changed very little, thus disproving the idea that marginal stability was enforced and calling into question the existing ITG theories. The focus of the work presented here is to analyze one of the discharges from this series in detail to try to understand this puzzling result.

We focus on TFTR discharge No. 44669 which is described in Table I and Fig. 1. This discharge was a "supershot" plasma with $\tau_{E} \approx 2.7 \tau_{E}^{L}$ and was heated by $14 \mathrm{MW}$ balanced neutral beam injection in a $1 \mathrm{MA}, 4.8 \mathrm{~T}$ target plasma with major radius $R=2.45 \mathrm{~m}$ and minor radius $a=0.80 \mathrm{~m}$. A deuterium pellet was injected at $t=4.50 \mathrm{sec}$ and penetrated only part way into the plasma, transientiy producing a flat density profile corresponding to very large values of the stability parameters $\eta_{i}$ and $\epsilon_{n}=L_{n} / R$. In the figures and tables, the letters $A$ and $B$ are used to designate the plasma state before and after the pellet injection. Figure 2 shows the radial profile of $\chi_{i}(r)$ obtained from the ion power balance analysis. As shown in Fig. $3, L_{T_{i}}$ did not change much (in fact, it dropped slightly), but because of the large rise in $L_{n}$, the stability limit for $L_{T_{i}}$ was greatly exceeded for a period of more than $20 \mathrm{msec}$, much longer than the growth time for ITG instabilities. There was little change in the thermal diffusivities inferred from the power balance during these transients in TFTR, in apparent contrast to the earlier experiments in Alcator-C and ASDEX, and in disagreement with the existing theories that predicted very large values of $\chi_{i}$ if $\eta_{i} \gg \eta_{\text {crit }}$.

To illustrate the magnitude of the disagreement between theory and experiment, Fig. 2 compares the measured $\chi_{i}(r)$ before and after the pellet perturbation with several different theories: the analytic toroidal formula of Biglari et al. ${ }^{7}$ (BDR), and the numerically derived slab formula of Hamaguchi and Horton. ${ }^{8}$ [We have replaced the factor $\left(1+\eta_{i}\right)$ that appeared in the original analytic formulas with the factor $\left(\eta_{i}-\eta_{\text {crit }}\right)$, a modification motivated by the desire to

TABLE I. TFTR perturbative transport discharges: shot number 44669 , TRANSP number $2200, r=0.3 \mathrm{~m}$.

\begin{tabular}{cll}
\hline \hline Time into discharge & $4.490 \mathrm{sec}$ & $4.525 \mathrm{sec}$ \\
\hline$n_{e}\left(\times 10^{19} \mathrm{~m}^{-3}\right)$ & 3.03 & 3.98 \\
$T_{e}(\mathrm{keV})$ & 5.71 & 4.07 \\
$T_{t}(\mathrm{keV})$ & 8.4 & 3.55 \\
$L_{n} / R$ & 0.17 & 1.18 \\
$L_{\tau} / R$ & 0.074 & 0.056 \\
$\eta_{t}$ & 2.3 & 21 \\
$T_{e} / T$, & 0.68 & 1.15 \\
$\alpha_{\mathrm{MHD}}$ & 0.36 & 0.29 \\
$\hat{s}$ & 0.75 & 0.7 \\
$q$ & 1.52 & 1.53 \\
\hline \hline
\end{tabular}
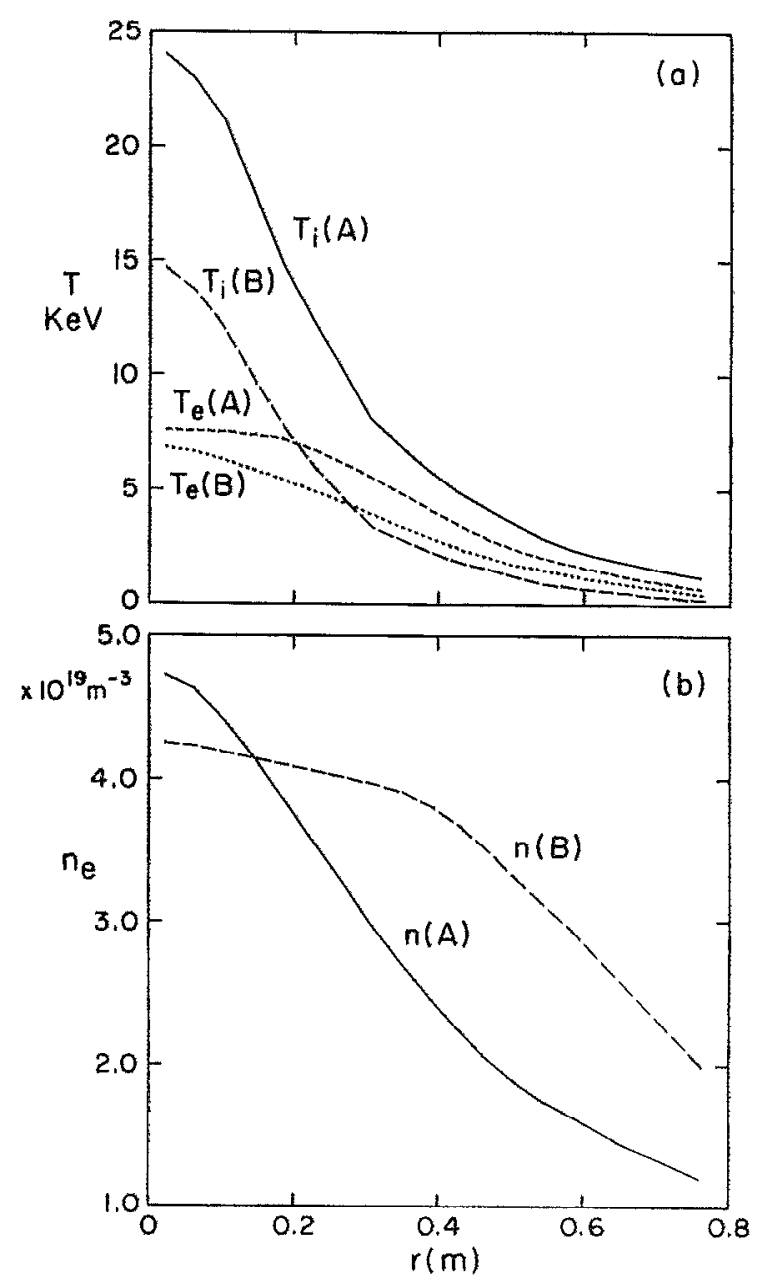

FIG. 1. Profiles of temperature and density for discharge 44669 in the A and B states.

have a reasonable transition to marginal stability and by the form of the Hamaguchi and Horton numerically derived $\chi_{i}$.] These previous theories predict $\chi_{i}^{\prime}$ 's that are 1-2 orders of magnitude too large in the core of the plasma.

Developing a complete first-principles theory of tokamak turbulence is not a realistic task in the foreseeable future because of a multitude of active processes in the tokamak plasmas. ${ }^{9}$ The standard picture of tokamak turbulence is based on drift-wave-type instabilities (including the $\eta_{i}$ mode and trapped electron modes) that generate small-scale $\mathbf{E} \times \mathbf{B}$ convective turbulence (although there is some uncertainty, both theoretically and experimentally, about whether small or large scale lengths dominate the transport). In order to make analytic progress and derive simple expressions for $\mathcal{X}_{i}$ and $\chi_{e}$, various approximations are made about the geometry (slab or toroidal), the dominant driving force (such as $\eta_{i}$ ), the mode structure, the collisionality, the nonlinear saturation mechanisms, and the nonlinear spectrum. The most complete formulas for $\mathcal{X}_{i}$ to date are based on parametrization of three-dimensional (3-D) nonlinear computer simulations using a two-component hydrodynamic description of the plasma.

The ITG theories shown in Fig. 2 were based on a num- 

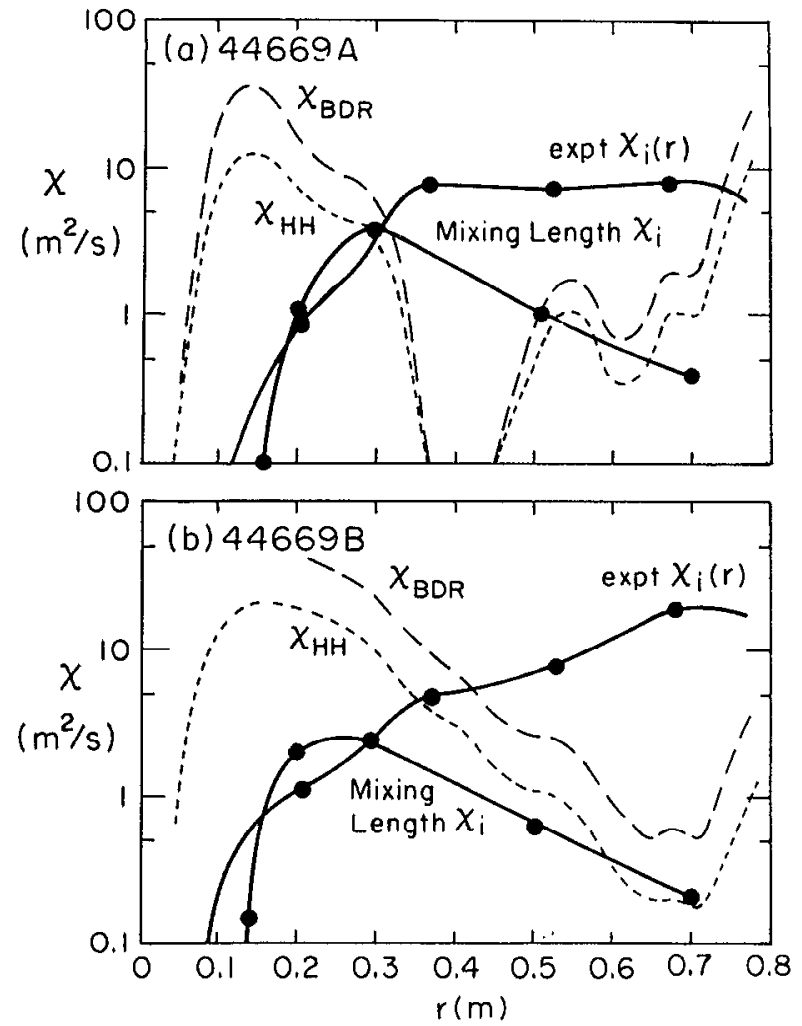

FIG. 2. Comparison of three theoretical models of ion thermal conductivity with experimental results for shot $44669 \mathrm{~A}$ and B states.

ber of simplifications, which caused them to predict a $\chi_{i}$ that is clearly too large. These theories were based on simplified fluid equations that did not adequately model finite-gyroradius effects or kinetic effects such as Landau damping (there is ongoing work to improve the fluid equations in this regard $^{10}$ and to use more complex fluid models ${ }^{11}$ ). More accurate kinetic (particle) simulations ${ }^{12}$ show that the actual growth rates and mode widths should be significantly smaller than given by the simplified fluid equations. Fortu-

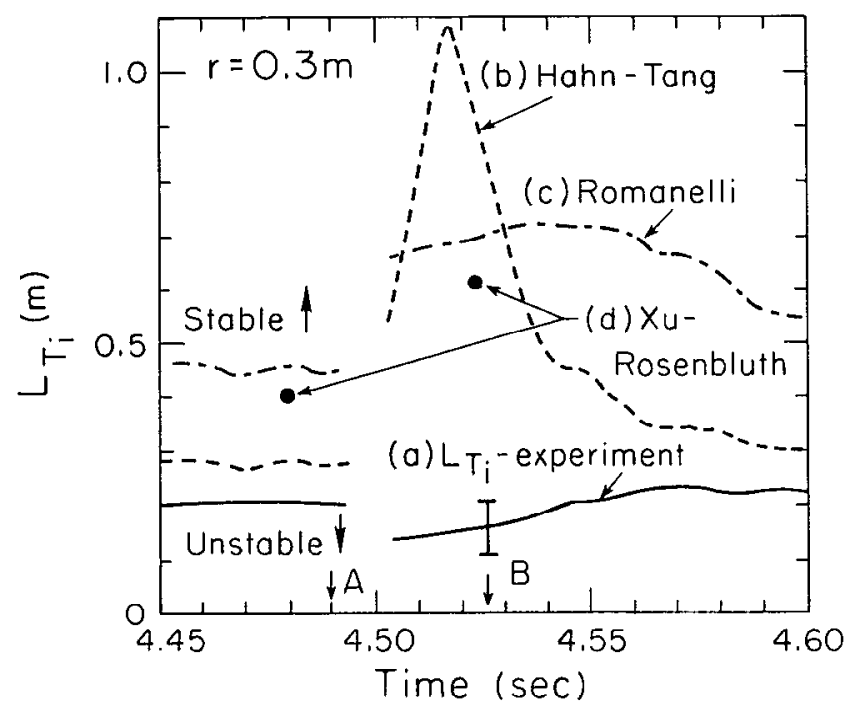

FIG. 3. Comparison of three theoretical models of critical temperature gradient length $\left(L_{T}\right)$ with experimental result at $r=0.3 \mathrm{~m}$ as a function of time. The reference times for states $\mathrm{A}$ and $\mathrm{B}$ are marked. nately, both fluid and kinetic simulations seem to support the mixing length theory and the scaling law analysis for the turbulent diffusivities based on the characteristics of the most unstable linear modes. Table II gives the ion and electron diffusives estimated from earlier theoretical works.

In this work, we show that a simple mixing length estimate applied to local kinetic theory, which incorporates toroidal and finite-gyroradius effects (missing from the previous theories) into a simple mixing length model is actually fairly consistent with the measured $\chi_{i}$ in the core of the plasma $(r<a / 3)$. However, the ITG mode appears to be too weak to explain the observed transport in the region $r>a / 3$, where either some other mode must be invoked or the simple mixing length estimate fails.

Some of the observed radial profile of $\chi_{i}(r)$ is obtained in the inner region when we use the local kinetic theory that retains the full particle-wave resonance effects from the magnetic curvature and $\nabla B$ drift and the parallel ion transit drift. The local kinetic analysis ${ }^{13}$ shows that the threshold for the ion-temperature-gradient-driven turbulence $\eta_{\text {crit }}$ is a function of $q(r)$. The $q$ value determines the connection length $q(r) R$ between the good and bad toroidal curvature regions as well as the ratio of the strength of the ion Landau resonance from $k_{\|} v_{\|}$to the grad- $B$ curvature drift resonance $\omega_{D}=k_{y} v_{D}\left(v_{1}^{2}, v_{\|}^{2}\right)$. Both these effccts work together to make the $\eta_{i}$ threshold higher at low $q$. Using this aspect of kinetic theory in the turbulence formulas for $\chi_{i}$ produces an increase of $\chi_{i}(r)$ with radius in the core region.

The present $\eta_{i}$ theories seem to be insufficient to explain the radial dependence of $\chi_{i}$ in the outer region. Here, we consider the possibilities of obtaining the observed increase of $\chi_{i}$ with $r / a$ by adding the effects of (i) the finite-beta drift wave magnetohydrodynamic (MHD) mode coupling, (ii) the slablike mode, or (iii) the trapped electron resonance, all of which are found to be inadequate. We also discuss other possible effects such as small-scale oscillations in the gradients or an unmeasured $\mathbf{E} \times \mathbf{B}$ poloidal shear flow as possible mechanisms for increasing the mixing width $\Delta X$ of the $\eta_{i}$ modes in the outer region.

The structure of the paper is as follows. In Sec. II, the

TABLE II. TFTR drift wave turbulent diffusivities: shot 44669 at $r=0.3$ m.

\begin{tabular}{|c|c|c|}
\hline & $t_{\mathrm{A}}=4.490$ & $t_{\mathrm{B}}=4.525$ \\
\hline $\mathcal{X i}_{i}^{\exp }$ & $=4 \mathrm{~m}^{2} / \mathrm{sec}$ & $3 \mathrm{~m}^{2} / \mathrm{sec}$ \\
\hline$D_{\mathrm{dw}}$ & $=\left(\rho_{s} / a\right)\left(c T_{e} / e B\right)$ & \\
\hline$\chi_{e}^{(1)}$ & $\begin{array}{l}=3.45 \mathrm{~m}^{2} / \mathrm{sec} \\
=a v_{e} c^{2} / q R^{2} \omega_{p c}^{2}\end{array}$ & $2.07 \mathrm{~m}^{2} / \mathrm{sec}$ \\
\hline$x^{(2)}$ & $\begin{array}{l}=2.58 \mathrm{~m}^{2} / \mathrm{sec} \\
=(a / R)^{1 / 2}\left(\rho_{c} c T / a e B\right)\end{array}$ & $1.66 \mathrm{~m}^{2} / \mathrm{sec}$ \\
\hline & $=1.97 \mathrm{~m}^{2} / \mathrm{sec}$ & $3.85 \mathrm{~m}^{2} / \mathrm{sec}$ \\
\hline$\chi_{i}^{\mathrm{HH}}$ & $=\left(\rho_{s} c T_{i} / L_{n} e B\right)\left(\eta_{i}-\eta_{i c}\right)$ & $\frac{\rho_{s} c T_{i}}{L_{T_{t}} e B} \exp \left(-4 \frac{T_{e} L_{r}}{T_{t} L_{s}}\right)$ \\
\hline$\chi_{i}^{\mathrm{BDR}}$ & $\begin{array}{l}\times \exp (-5 \hat{s})=9.1 \mathrm{~m}^{2} / \mathrm{sec} \\
=(q / \hat{s}) \omega_{*_{e}} \rho_{i}^{2}\left(1+\eta_{i}\right)\end{array}$ & $=12 \mathrm{~m}^{2} / \mathrm{sec}$ \\
\hline$\gamma_{\mathrm{KH}}^{\mathrm{KH}}$ & $\begin{array}{l}=8.1 \mathrm{~m}^{2} / \mathrm{sec} \\
=\gamma / k^{2}\end{array}$ & $5.0 \mathrm{~m}^{2} / \mathrm{sec}$ \\
\hline $\mathcal{X}_{i, \text { Local-Vlasov }}$ & $\begin{array}{l}=\gamma_{m} / k_{m} \\
=5.2 \mathrm{~m}^{2} / \mathrm{sec}\end{array}$ & $2.4 \mathrm{~m}^{2} / \mathrm{sec}$ \\
\hline
\end{tabular}


transport in TFTR discharge 44669 is compared with various models of the $\eta_{i}$ mode. First, a detailed analysis is given from the local kinetic theory. Then the clcctrostatic and electromagnetic ballooning analysis is followed to complement the local analysis. Also, the sheared slab model is discussed for completeness. In Sec. III, in the attempts to solve the disagreement problem of the radial profile of $\chi_{i}$ at the outer region, various stability effects such as steeper edge gradients and trapped electron resonance are discussed. Finally, in Sec. IV, the conclusions are summarized.

\section{DRIFT WAVE STABILITY ANALYSIS}

\section{A. Local electrostatic kinetic analysis}

We begin the analysis of the discharge by determining the unstable spectrum from local, electrostatic stability theo- ry using the parameters from Table I. For Maxwellian velocity distributions, the electrostatic dispersion relation is

$$
D_{\mathrm{ES}}(\mathbf{k}, \omega)=\sum_{j} \frac{n_{j} e_{j}^{2}}{T_{j}}\left(1-\left\langle\frac{\omega-\omega_{* j}(\epsilon)}{\omega-\omega_{D_{j}}-k_{\|} u_{\|}} J_{0}^{2}\right\rangle\right)
$$

The $j$ summation is over electrons, ions, and impurities. In Sec. III B, we briefly consider the effect of the carbon impurities on the stability. When only the thermal ions are taken as dynamical with the electrons and beam ions as adiabatic, the dispersion relation (1), in the standard dimensionless units, reduced to

$$
D_{\mathrm{ITG}}\left(k_{y}, k_{\|}, \omega\right)=D_{a}-\int_{0}^{\infty} \int_{-\infty}^{+\infty} \frac{\left\{\tau \omega-k_{y}\left[1+\eta\left(v^{2} / 2-3 / 2\right)\right]\right\} J_{0}^{2}\left(k_{1} v_{1} / \tau^{1 / 2}\right) e^{-v^{2} / 2}}{\tau \omega-k_{y} \epsilon_{n}\left(\frac{1}{2} v_{1}^{2}+v_{\|}^{2}\right)-k_{\|} v_{\|} \tau^{1 / 2}} \frac{v_{1} d v_{1} d v_{\|}}{(2 \pi)^{1 / 2}}
$$

where the adiabatic response $D_{a}$ is given by

$D_{a}=1+\frac{n_{i} T_{e}}{n_{e} T_{i}}+\frac{n_{b} T_{e}}{n_{e} T_{b}}+\frac{n_{z} Z^{2} T_{e}}{n_{e} T_{z}}\left[1-I_{0}\left(b_{z}\right) e^{-b_{z}}\right]$.

In Eq. (3), we include a hydrodynamic impurity ion contribution where $b_{z}=k_{1}^{2} \rho_{z}^{2}$, and we assume the impurity drift frequency $\omega_{*_{z}}=0$. The adiabatic response reduces to the usual $D_{a} \cong 1+T_{e} / T_{i}$ of ideal ITG mode theory when the impurity and beam densities are sufficiently low. The usual dimensionless parameters in Eq. (2) are $\epsilon_{n}=r_{n} / R$ with $r_{n}=L_{n}=-(\partial \ln n / \partial r)^{-1}$, and $\tau=T_{e} / T_{i}$ and the fluctuation variables $k_{1}, k_{\|}$, and $\omega$ are normalized to $\rho_{s}=c_{s} / \omega_{c i}$, $r_{n}$, and $r_{n} / c_{s}$ with $c_{s}=\left(T_{e} / m_{i}\right)^{1 / 2}$.

The marginal stability analysis of the dispersion relation in (2) gives the condition $\eta_{i}>2 / 3$ and

$$
\epsilon_{T}=\epsilon_{n} / \eta<0.7 / D_{a}
$$

for the threshold of instability. The toroidal threshold condition (4) is derived by Dominguez and Waltz ${ }^{14}$ and Horton et $a l .{ }^{15}$ and is often called the Romanelli ${ }^{16}$ condition

$$
\left(\frac{L_{T_{i}}}{R}\right)_{\text {crit }}=\frac{0.7}{1+T_{i} / T_{e}},
$$

since Romanelli emphasized its practical importance. Clearly, the role of magnetic shear is considered subdominant when applying conditions (4) or (5) since the formulas are independent of $s$.

In the case where the magnetic shear length $L_{s}(r)$ is relatively short $\left(L_{s}<R\right)$, the results of marginal stability from the sheared-slab eigenmode analyses in the flat density profile limit are given by Hahm and Tang ${ }^{17}$

$$
\left(\frac{L_{T_{i}}}{L_{s}}\right)_{\text {crit }}^{-1}=\frac{3}{2} \sqrt{\frac{\pi}{2}}\left(1+\frac{T_{i}}{T_{e}}\right)(2 l+1)
$$

Here, $l=0,1,2, \ldots$ is the radial mode number.
Both stability conclusions (5) and (6) state that, for fixed scale lengths, the system is stable when $T_{e} / T_{i}$ falls below a critical value $\left(T_{e} / T_{i}\right)_{\text {crit }}$. In both the $\mathrm{A}$ and $\mathrm{B}$ states, we find that the plasma is well above this critical value $\tau_{\text {crit }}$, as shown in Fig. 3. As a function of $\tau=T_{e} / T_{i}$, the growth rate first increases as $\tau-\tau_{\text {crit }}$ and then decreases as $(1 / \tau)^{1 / 2}$ for $\tau \gg \tau_{\text {crit }}$. In the hydrodynamic approximation, Hamaguchi and Horton ${ }^{8}$ give

$$
\gamma_{k} \cong\left|\omega_{*}\right|\left[\left(\frac{\eta_{i} S}{2 \tau}\right)^{1 / 2}-\frac{S}{2}\left(1+\frac{3}{4} \frac{\Gamma}{\tau}\right)\right]
$$

with $\eta_{\text {crit }}$ in the flat density limit given by $\eta_{\text {crit }}=\left(S T_{e} / 2 T_{i}\right)\left[1+\frac{3}{4}(\Gamma / \tau)\right]^{2}$, where $\Gamma$ is the ideal gas constant and $S=L_{n} / L_{s}$. The maximum $\gamma_{k}(\tau)$ occurs at $\tau_{m}=\Gamma^{2} S / \eta$ with the value of $\gamma_{m}=\frac{1}{3}\left|\omega_{\bullet_{e}}\right|\left(\eta_{i} / \Gamma-\frac{3}{2} S\right)$. The local stability analysis indicates that the experiment is in the regime of $\gamma_{k}$ decreasing with increasing $\tau$. The comparison of the stability conditions on $L_{T}$ for the transport discharge is given in Fig. 3 showing that the plasma is unstable to both criteria at all times. The critical values for $L_{R_{1}}$ obtained by the integral equation analysis by $\mathrm{Xu}$ and Rosenbluth ${ }^{18}$ are also shown to be close to the value from the Romanelli formula given by the curve labeled (c).

While the threshold formulas given in Eqs. (4)-(7) are useful, a simpler and more direct picture of the stability of the system is obtained by finding all the local eigenmodes $\omega_{k}(\mathbf{r}), \gamma_{k}(\mathbf{r})$ from the Vlasov dispersion relation. The sheared slab and ballooning modes may be viewed as certain linear superpositions of these local modes that form longlived states in the inhomogeneous system. We calculate such local solutions both varying the radial position and the poloidal angle to obtain a description of the stability of the system before and after the pellet injection.

In Fig. 4, we show the spectrum of $\gamma\left(k_{y}, k_{\|}, r\right)$ computed from Eq. (2) (with $n_{b}=n_{z}=0$ ) before (state A) and during the flat density profile perturbation (state $B$ ). 
A $v_{i 0} / R=2.4 \times 10^{5} \mathrm{~s}^{-1}$
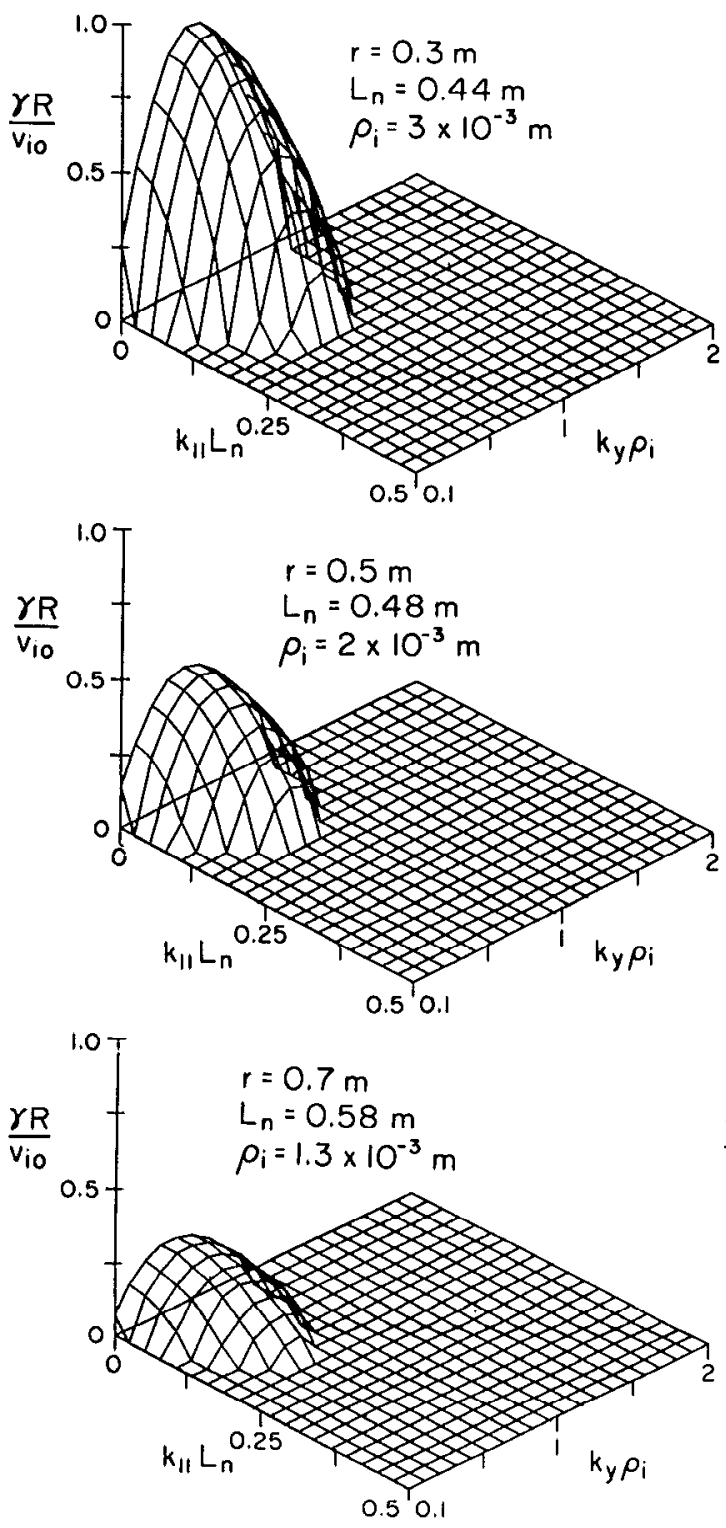

B $v_{i 0} / R=1.56 \times 10^{5} \mathrm{~s}^{-1}$
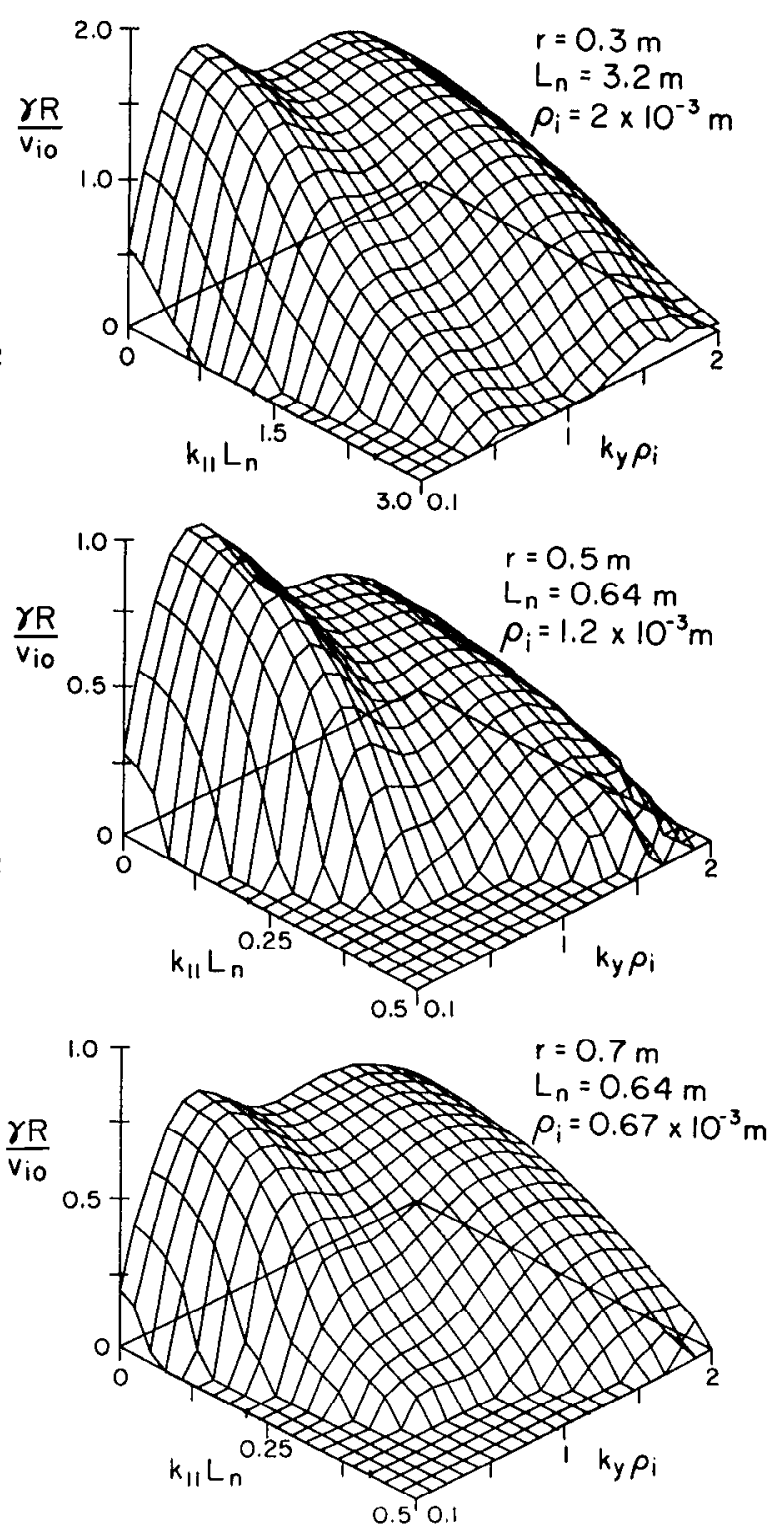

FIG. 4. The local kinetic growth rates in the wave-number domain for the shot $44669 \mathrm{~A}$ and B states.

The growth rates in Fig. 4 are normalized by $v_{0} / R\left(2.4 \times 10^{5} \mathrm{sec}^{-1}\right)$ for state $\mathrm{A}$ and $v_{i 0}^{\prime} / R$ $\left(1.56 \times 10^{5} \mathrm{sec}^{-1}\right)$ for state $\mathrm{B}$, where $v_{i 0}$ and $v_{i 0}^{\prime}$ are the ion thermal velocity $\left(T_{i} / m_{i}\right)^{1 / 2}$ at $r=0.3 \mathrm{~m}$ in the states $\mathrm{A}$ and $B$, respectively. We see that, in both states, there is a large spectrum of unstable wave numbers. The principal effect of the large increase in $\eta_{i}$ is to destabilize the modes with $k_{1} \rho_{i}>1$. In fact, in the flat profile state, a secondary local maximum is produced at $k_{\perp} \rho_{i} \sim 1.5$, which is a stable region before the density flattening. However, these short-wavelength modes may not have a significant effect on the transport because nonlinear 3-D simulation studies ${ }^{8,12}$ support the theoretical picture that the correct measure of the transport is $\gamma / k_{x}^{2} \sim \gamma / k_{y}^{2}$, since the turbulent states are found to be isotropic $\left\langle k_{x}^{2}\right\rangle \sim\left\langle k_{y}^{2}\right\rangle$ with the peak of the $k_{y}$ spectrum only slightly down shifted from the $k_{y}$, which maximizes $\gamma_{k_{y}}$ depending on $\eta_{i}, \epsilon_{n}$, and $s$. The secondary instability giving rise to the isotropization in $k_{x}-k_{y}$ is analyzed in Cowley et al. ${ }^{19}$

The long-wavelength modes $\left(k_{\perp} \rho_{i}<1\right)$ have the maximum dimensionless growth rate $\gamma_{m} L_{n} / c_{s}$ increasing with the increase of $\eta_{i}$. However, during the perturbation, the value of $L_{n}$ and the ion temperature $T_{i}$ change strongly (see Fig. 1). Thus, returning to the actual growth rate, we find that, at $r=0.3 \mathrm{~m}$, the maximum growth rate $\gamma_{m}$ is slightly increased from $2.1 \times 10^{5} \mathrm{sec}$ to $2.6 \times 10^{5} \mathrm{sec}$ and its location shifts from $k_{m}=2 \mathrm{~cm}^{-1}$ to $k_{m}=3.2 \mathrm{~cm}^{-1}$. Fluid turbulence simulations imply that the expected turbulent trans- 
port is then $\chi_{i}(r=0.3 \mathrm{~m}, \mathrm{~A})=\gamma_{m} / k_{m}^{2}=5.2 \mathrm{~m}^{2} / \mathrm{sec}$ compared with $\chi_{i}(r=0.3 \mathrm{~m}, \mathrm{~B})=2.4 \mathrm{~m}^{2} / \mathrm{sec}$. While numerous parameters change from the $\mathrm{A}$ to $\mathrm{B}$ states of the discharge, the dominant change for the $\chi_{i}$ value at $r=0.3 \mathrm{~m}$ is the decrease of the ion temperature from 8.4 to $3.55 \mathrm{keV}$. In Table III, the mixing length values of $\chi_{i}$, obtained from the local Vlasov stability analysis described by Eq. (1), are given at various radii for the $\mathrm{A}$ and $\mathrm{B}$ states.

From the local analysis, we also see that the degree to which the local parameters are in the toroidal regime of small $k_{\|} v_{T} / \omega_{D i}$ in contrast to the sheared slab regime with $x=k_{\|} v_{T} / \omega_{D i}>1$ is an important influence on the stability of the ITG modes. As analyzed in detail in Kim and Horton ${ }^{13}$ and Dominguez and Rosenbluth, ${ }^{20}$ this dependence on $k_{\|\|} v_{T} / \omega_{D i} \sim 1 / q k_{y} \rho$ taking $k_{\|}=1 / q R$ gives a $q$ dependence to the growth rate $\gamma_{\max }(q)$, the threshold $\eta_{\text {crit }}$, and the associated transport. In Fig. 2, we plot the results given in Table III labeled as "mixing length $\chi_{i}$," and compare with experimental results. The kinetic mixing length $\chi(r)$ in Fig. 2 has a radial dependence that is in considerably better agreement with the experimental profile at the inner region of $r<a / 3$ than the $\chi_{i}^{\mathrm{HH}}(r)$ and $\chi_{i}^{\mathrm{BDR}}(r)$ formulas. However, at the outer region of $r>a / 2$, there is still significant disagreement in the radial dependence. The unfavorable radial dependence arises from the rapid decrease of $T_{i}(r)$, which overcomes the increase of $\chi_{i}$ with $q$ at fixed $T_{i}$.

\section{B. Electrostatic ballooning mode stability analysis}

Here, we analyze the stability of the system to the electrostatic ballooning mode equation assuming that the ion acoustic dynamics $k_{i \mid}^{2} c_{s}^{2} / \omega^{2}$ can be expanded to first order in the kinetic response functions in Eq. (1). The ballooning eigenmode equation gives the proper averaging over the spectrum of parallel wavelengths that occurs at each $k_{y} \rho_{s}$ and radius. The change in the eigenvalues from the local value given in Sec. II A occurs from the ion acoustic wave propagation along the magnetic field lines. Many basic studies of the drift wave ballooning mode equation for the $\eta_{i}$ mode problem from numerous groups are available as reviewed in Horton. ${ }^{9}$ Here, we give the ballooning mode equation used in the study with minimal explanation for the present application. The electrostatic mode equation ${ }^{21}$ is

$$
\begin{aligned}
& {\left[\left(1-\frac{\omega_{*_{i}}}{\omega}\right) \Gamma_{0}-\frac{\omega_{*_{i}}}{\omega} \eta_{i}\left[\Gamma_{0}+b\left(\Gamma_{1}-\Gamma_{0}\right)\right]\right] \frac{\epsilon_{n}^{2}}{q^{2} \omega^{2}} \frac{\partial^{2} \phi}{\partial \theta^{2}}} \\
& \quad+\{1+\tau[1-P(\theta)]\} \phi(\theta)=0
\end{aligned}
$$

with the boundary conditions $\phi(\theta \rightarrow \infty) \rightarrow 0$ sufficiently rap-

TABLE III. Mixing length $\chi$, from local Vlasov theory: shot 44669, using $\gamma_{\max }$ at $k_{\|}=1 / q R$, and $\left\langle k_{x}^{2}\right\rangle=\left\langle k_{y}^{2}\right\rangle=k_{y}^{2}\left(\gamma_{\max }\right)$.

\begin{tabular}{ccc}
\hline \hline$r$ & Before injection & After injection \\
\hline $0.15 \mathrm{~m}$ & $0.0 \mathrm{~m}^{2} / \mathrm{sec}$ & $0.16 \mathrm{~m}^{2} / \mathrm{sec}$ \\
$0.3 \mathrm{~m}$ & $5.2 \mathrm{~m}^{2} / \mathrm{sec}$ & $2.4 \mathrm{~m}^{2} / \mathrm{sec}$ \\
$0.5 \mathrm{~m}$ & $1.2 \mathrm{~m}^{2} / \mathrm{sec}$ & $0.7 \mathrm{~m}^{2} / \mathrm{sec}$ \\
$0.7 \mathrm{~m}$ & $0.4 \mathrm{~m}^{2} / \mathrm{sec}$ & $0.21 \mathrm{~m}^{2} / \mathrm{sec}$ \\
\hline \hline
\end{tabular}

idly for $\left\langle k_{x}^{2}\right\rangle \propto s^{2} k_{y}^{2} s_{-\infty}^{\infty} \theta^{2} \phi^{2} d \theta$ to exist. In Eq. (8), the kinetic response function $P$, given in Eq. (A3) in the Appendix, is a function of $\theta$ and vanishes as $1 / \theta^{2}$ for large $\theta$. The local perpendicular and parallel wave numbers are given by

$$
\begin{aligned}
& k_{\perp}^{2} \rho_{i}^{2}=\left(k_{y}^{2} \rho_{s}^{2} / \tau\right)\left(1+s^{2} \theta^{2}\right), \\
& k_{\|}=-\frac{i}{q R} \frac{1}{\phi} \frac{\partial \phi}{\partial \theta},
\end{aligned}
$$

and the local grad- $B$ and curvature drift frequency is

$\omega_{D i}=-\epsilon_{n} k_{y} \rho_{s} \frac{m_{i}}{T_{i}}\left(\frac{1}{2} v_{1}^{2}+v_{\|}^{2}\right)(\cos \theta+s \theta \sin \theta)\left(\frac{c_{s}}{L_{n}}\right)$

for low-beta, circular flux surfaces.

The ballooning mode equation ( 8 ) has a series of eigenfunctions describing the normal modes of the plasma. As in the sheared slab, we designate the $l$ th mode by $\phi_{k_{y} l}(\theta)$ and order the modes with increasing oscillations with $l=0,1,2, \ldots$. Important measures of the characteristics of the modes are given by the integral width $\Delta \theta_{I}$ and the differential width $\Delta \theta_{D}$. We also define the mean value of the expansion parameter $P_{\|}=\overline{k_{\|}^{2}} v_{i}^{2} / \omega^{2}$ used in obtaining the differential equation ( 8 ) from the integral mode equation. The definitions of the $\phi(\theta)$ measures are

$$
\begin{aligned}
& \Delta \theta_{I}^{2}=\frac{\int_{0}^{\infty} d \theta \theta^{2} \phi^{2}(\theta)}{\int_{0}^{\infty} d \theta \phi^{2}(\theta)}, \\
& \frac{1}{\Delta \theta_{D}^{2}}=\frac{\int_{0}^{\infty} d \theta\left[\phi^{\prime}(\theta)\right]^{2}}{\int_{0}^{\infty} d \theta \phi^{2}(\theta)} .
\end{aligned}
$$

The ballooning mode wave function $\phi(\theta)$ gives a ballooning mode radial width $\Delta X_{b}$ and $k_{x}$ given by

$$
\left\langle k_{x}^{2}\right\rangle=\Delta X_{b}^{-2}=k_{y}^{2} s^{2} \operatorname{Re}\left(\Delta \theta_{I}^{2}\right),
$$

with the subscript $b$ for ballooning. This ballooning mode width and the associated $\gamma /\left\langle k_{x}^{2}\right\rangle$ has been estimated theoretically in Horton et $a l^{22}$ and Dominguez and Rosenbluth. ${ }^{20}$ The resulting diffusivities are similar to that given as $\chi^{\mathrm{BDR}}$ in Table II. The ballooning $\chi_{i}$ varies inversely proportionally with shear $s=r q^{\prime} / q$ and proportionally to $q$, which, for fixed $T_{i}$, gives a $\chi_{i}$ that increases with $r / a$.

The expansion parameter for measuring the strength of the ion acoustic wave effect is $\left\langle k_{\|}^{2}\right\rangle v_{i}^{2} / \omega^{2}$, which, in the dimensionless variables, is given by

$$
P_{\|}=\frac{T_{i}}{T_{e}} \frac{\epsilon_{n}^{2}}{q^{2}} \frac{1}{|\omega|^{2}\left|\Delta \theta_{D}^{2}\right|},
$$

and the validity of the differential Eq. (8) requires that $P_{\|}<1$. Now, using Eq. (8), we consider the ballooning stability of the modes identified as most dangerous from the local stability analysis in Sec. II A.

For each radial position in Table III, we have carried out the integration of the ballooning mode equation in Eq. (8) to find the lowest-order eigenmodes and eigenvalues. First of all, the ballooning mode analysis shows that there are two important fast growing modes: One is peaked at $\theta=0$, which we call the outside mode and one peaked in the region $\theta=\pi / 3$ to $2 \pi / 3$, which we call the top/bottom mode since the peak intensity is somewhere in those regions rather than on the outside. The shapes of the eigenfunctions of these 
modes are shown in Fig. 5 for $r=0.3 \mathrm{~m}$ before and after injection. Generally, the growth rates of the top/bottom modes are about one-half that of the outside mode and the frequency of the top/bottom mode is 1.5 times greater than the outside mode. Both frequencies are generally somewhat above the local kinetic transit frequency $k_{\|} v_{i}$ obtained with $k_{\|}=1 / q R$. We find that the growth rates of the outside mode are close to those obtained with the local kinetic theory.

We have computed the moments of the wave functions defined in Eqs. (9) and (10) to determine the expansion parameter $P_{\|}$and radial mode with $\Delta X_{b}$. We find that, even though the condition $P_{\|}<1$ is marginally satisfied over most radii, the outside mode growth rates are nearly equal to the local kinetic results. The top/bottom mode has a larger $P_{\|}$ than the outside mode, and thus is closer to the slab mode. The mode widths $\Delta X_{b}$ 's appear to be larger than $\rho_{s}$ over all radii before and after the discharge, so that the diffusivity estimate based on $\gamma \Delta X_{b}^{2}$ is somewhat larger than the isotropic turbulence mixing length $\Delta X_{\mathrm{ml}}=k_{y}^{-1}$. For example, for $44669 \mathrm{~A}$ and $r=0.3 \mathrm{~m}$, we obtain $\Delta X_{b} / \rho_{s}=2.9$ with $P_{\|}=0.20$ so that $\chi_{i}^{b}=6.8 \mathrm{~m}^{2} / \mathrm{sec}$ compared with 5.2 $\mathrm{m}^{2} / \mathrm{sec}$ from $\Delta X_{\mathrm{ml}}=1 / k_{y}\left(\gamma_{\max }\right)$. It must be emphasized that the mode width $\Delta X_{b}$ from Eq. (11) corresponds to the linear regime. From the nonlinear studies, ${ }^{8,9,12,19}$ however, we recognize that the nonlinear saturation forces the formation of approximately circular vortices so that the proper mixing length is $k_{y}^{-1}$ when $k_{y} \Delta X_{b}>1$. Now, with this consideration the results from the ballooning mode calculation using $\Delta X_{\mathrm{ml}}=1 / k_{y}\left(\gamma_{\max }\right)$ are given in Table IV. Comparing Table III and Table IV, we see that the ballooning mode analysis agrees quite well with the local kinetic analysis.

The expansion used to derive Eq. (8) is only valid if $P_{\| 1} \ll 1$, which is marginally satisfied for our parameters. Calculating moments of the eigenfunction and using Eqs. (10)
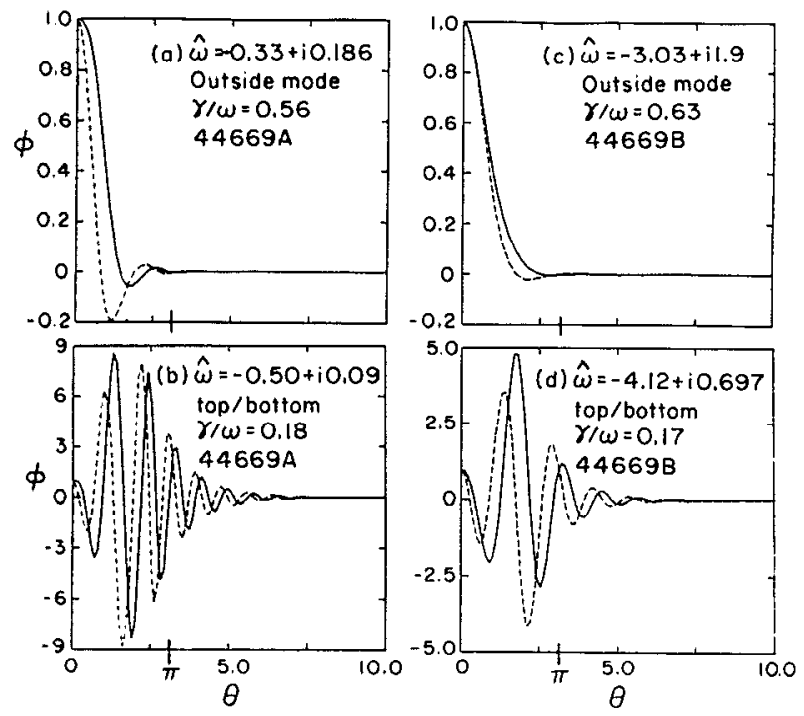

FIG. 5. The shapes of the kinetic ballooning eigenfunctions for both the outside and top/bottom modes at $r=0.3 \mathrm{~m}$ for $44669 \mathrm{~A}$ and B states.
TABLE IV. Mixing length $\chi_{i}$ from electrostatic ballooning equation: shot 44669, using $\chi_{i}=\gamma_{m} \Delta X_{\mathrm{m} 1}^{2}$ and $\Delta X_{\mathrm{ml}}^{2}=k_{y}{ }^{-2}\left(\gamma_{m}\right)$.

\begin{tabular}{ccc}
\hline$r$ & Before injection & After injection \\
\hline $0.15 \mathrm{~m}$ & $0.19 \mathrm{~m}^{2} / \mathrm{sec}$ & $0.13 \mathrm{~m}^{2} / \mathrm{sec}$ \\
$0.3 \mathrm{~m}$ & $5.1 \mathrm{~m}^{2} / \mathrm{sec}$ & $2.2 \mathrm{~m}^{2} / \mathrm{sec}$ \\
$0.5 \mathrm{~m}$ & $1.23 \mathrm{~m}^{2} / \mathrm{sec}$ & $0.68 \mathrm{~m}^{2} / \mathrm{sec}$ \\
$0.7 \mathrm{~m}$ & $0.37 \mathrm{~m}^{2} / \mathrm{sec}$ & $0.21 \mathrm{~m}^{2} / \mathrm{sec}$ \\
\hline
\end{tabular}

and (12), we find that $P_{\|}=0.2$ for the outside mode at $r=0.3 \mathrm{~m}$ at time $A$. Nevertheless, the growth rates for the outside mode from the ballooning equation, Eq. (8), are close $(\Delta \gamma / \gamma \leqslant 20 \%)$ to the growth rates found from the local dispersion relation, Eq. (2), which made no expansion in $P_{\|}$. This gives some confidence that this calculation is approximately correct, at least for the outside mode, which is primarily driven by toroidal curvature. The good comparison between the two approaches is due in part to the use of $k_{\|}=1 / q R$ in the local theory, which agrees well with average $k_{\|}$calculated by our ballooning equation. However, it is possible that a more complete ballooning calculation that does not depend on a small $P_{\|}$ordering might produce a different spectrum of $k_{\|}$'s. This may be important because part of the drop of the theoretical $\chi_{i}$ near the axis is due to the stabilization of the ITG mode at large $k_{\|}$because of the assumed $k_{\|}$dependence on $1 / q$, which is getting large near the axis.

An interesting area for future work would be to apply a more complete ballooning mode calculation that does not rely on this small $P_{\|}$approximation. In fact, comparing Fig. 14 of Ref. 18 (which makes no assumptions about $k_{\|}$or $P_{\|}$) with Fig. 4 of Ref. 20 (which makes $P_{\|} \ll 1$ and $k_{\|}=1 / q R$ approximations), one finds that the stabilization of ITG modes at low $q$ is overstated by the $k_{\|}=1 / q R$ approximation.

While our expansions may be marginally acceptable for the outside mode, they are not useful for the top/bottom mode for which we find $P_{\|} \approx 7$ and $k_{\|} \approx 4 / q R$. The top/bottom mode is not affected much by the toroidal curvature drive. We will analyze this slablike mode with a theory that is valid for general $P_{\|}$in Sec. II D.

\section{Electromagnetic ballooning analysis}

To complete the stability analysis of the discharges, we consider the electromagnetic ballooning mode analysis. Here, again, there are many works giving the details of the theoretical analysis including, but not limited to the ones of Cheng, ${ }^{23}$ Tang et al., ${ }^{24}$ Hong et al., ${ }^{21}$ and Dominguez and Moore. $^{25}$

Using the $\psi(\theta)$ potential for $A_{\|}$such that $E_{\|}=-i k_{\|}(\phi-\psi)$, we obtain the electromagnetic mode equation

$$
\begin{gathered}
{\left[\frac{\omega_{A}^{2}}{\omega^{2}} \frac{\partial}{\partial \theta} k_{1}^{2} \frac{\partial}{\partial \theta}+\left(1-\frac{\omega_{*_{e}}}{\omega}\right)\left(1-\frac{\omega_{D e}}{\omega}\right)\right.} \\
\left.-\frac{\left(1-\omega_{*_{e}} / \omega\right)^{2}}{D_{\mathrm{ES}}(\omega, k, \theta)}\right] \psi=0
\end{gathered}
$$


valid for $P_{\|}<1$. Some details of the derivation of Eq. (13) are given in the Appendix. The $s-\alpha$ equilibrium model is used as a simple approximation for the equilibrium. In this model, the local wave number and drift frequency become

$$
\begin{aligned}
& k_{1}^{2}=k_{y}^{2} \rho_{s}^{2}\left[1+(s \theta-\alpha \sin \theta)^{2}\right], \\
& \omega_{D i}=\bar{\omega}_{D i}[\cos \theta+(s \theta-\alpha \sin \theta) \sin \theta],
\end{aligned}
$$

giving the reversal of the magnetic shear for $\alpha>1$. Here, $\alpha=-2 R q^{2} d \beta / d r$, with $\beta$ containing all the pressure components, and reaches a maximum value of 0.4 in $44669 \mathrm{~A}$ and B.

In Table $V$, we show the growth rates and real frequencies obtained from the local electromagnetic dispersion relation at various radii for the discharge 44669 before and during the density modification. The spectrum of modes obtained from Eq. (13) contains both the local electrostatic toroidal ITG mode, which has $\omega_{A}^{2} / \omega^{2} \gg 1$ and thus satisfies Eq. (13) by having the electrostatic dispersion relation $D_{\mathrm{ES}}(\omega, k, \theta) \approx 0$, and the high-frequency kinetic FLR-MHD mode rotating with

$$
\omega \simeq \omega_{*_{i}}\left(1+\eta_{i}\right) .
$$

The kinetic finite Lamar radius (FLR)-MHD mode is destabilized by the $\omega=\omega_{D i}$ drift resonance below the MHD beta limit $\alpha_{\text {crit }}{ }^{21,23,24}$ The $\alpha$ is sufficiently below $\alpha_{\text {crit }}$ in these discharges that kinetically modified FLR-MHD mode is a real oscillation with $\omega=-5.09 \times 10^{6} \mathrm{sec}^{-1}$ at $r=0.3 \mathrm{~m}$ and $k_{y} \rho_{s}=0.5$. Table $\mathrm{V}$ shows that the electromagnetic effect on the electrostatic toroidal ITG mode is stabilizing. For each radius, there are two modes with their polarization given in the right-hand column, where $E_{\|}=0$ at $\psi / \phi=1$ and $\delta B_{r}=0$ at $\psi / \phi=0$. On the other hand, to obtain the MHD $\left(k_{y} \rho \rightarrow 0\right)$ beta limit, the mode equation (13) is expanded in the fluid limit to obtain

$$
\begin{gathered}
\frac{\epsilon_{n}^{2}}{q^{2}} \frac{2}{\beta_{e}} \frac{\partial}{\partial \theta} k_{1}^{2} \frac{\partial}{\partial \theta} \psi+\left[\omega\left[\omega-\omega_{*_{i}}\left(1+\eta_{i}\right)\right] k_{1}^{2}(\theta)\right. \\
\left.+\omega_{*_{e}} \omega_{D e}(\theta)\left(1+\eta_{e}+\frac{1+\eta_{i}}{\tau}\right)\right] \psi=0 .
\end{gathered}
$$

For 44669 A, Fig. 6(a) shows the shape of an eigenfunction of Eq. (15) for $r=0.3 \mathrm{~m}, k_{y} \rho_{s}=0.5\left(k_{y}=2.2 \mathrm{~cm}^{-1}\right)$ before injection, where the eigenvalue is $\omega=-2.66 c_{s} / L_{n}$ $\left(3.13 \times 10^{5} \mathrm{sec}^{-1}\right)$ compared with $\omega_{*_{i}}\left(1+\eta_{i}\right)$ $=-2.55 c_{s} / L_{n} \quad\left(3.01 \times 10^{6} \quad \mathrm{sec}^{-1}\right) \quad$ and $\omega_{A}=v_{A} / q R=3.06 c_{s} / L_{n} \quad\left(3.6 \times 10^{6} \quad \mathrm{sec}^{-1}\right) \quad$ using $c_{s} / L_{n}=1.18 \times 10^{6} / \mathrm{sec}$. The ballooning mode width is $\Delta X_{b}=0.342 \rho_{s}$. This compares well with kinetic modified (FLR)-MHD eigenmode given by Eq. (13) with $\omega=-2.87 c_{s} / L_{n}\left(3.38 \times 10^{6} \mathrm{sec}^{-1}\right)$ and mode width $\Delta X_{b}=0.342 \rho_{s}$ at the same position.

In Fig. 6(b) the $\eta_{i}$-mode branch of the same EM equation is found by solving Eq. (13) with the electrostatic eigenmode as the first-trial function. The electromagnetically modified $\eta_{i}$-mode solution has $\omega=(-0.277+i 0.0272)$ $\times\left(c_{s} / L_{n}\right)$ or $(-32.7+i 3.21) \times 10^{4} \mathrm{sec}^{-1}$ eigenvalue and the ballooning mode width $\Delta X_{b}=0.690 \rho_{s}$. The electromagnetic modification has reduced $\gamma$ and the estimated $\chi_{i}$.

\section{Electromagnetic integral equation analysis in sheared slab}

In Secs. II A and II B, the ballooning mode analysis was made by expanding the parallel ion motion in the small $\left|k_{\|}^{2} v_{i}^{2} / \omega^{2}\right|$ limit. Here, we study the slablike branch, called the top/bottom mode in Fig. 5, from another theoretical description taking into account $\omega \sim k_{\|} v_{\text {, }}$ for completeness. For the slablike mode, we use the integral equation code of Dong et $a .^{26}$ in the electromagnetic regime with nonadiaba-

\begin{tabular}{|c|c|c|c|c|c|c|c|}
\hline $\begin{array}{c}r \\
(\mathrm{mI})\end{array}$ & $\begin{array}{c}\omega_{A} \\
\left(10^{s} / \mathrm{sec}\right)\end{array}$ & $\begin{array}{c}\omega \\
\left(10^{5} / \mathrm{sec}\right)\end{array}$ & $\begin{array}{c}\gamma \\
\left(10^{5} / \mathrm{sec}\right)\end{array}$ & $\begin{array}{c}k_{\|} \\
(1 / \mathrm{m})\end{array}$ & $\begin{array}{c}k_{1} \\
(1 / \mathrm{cm})\end{array}$ & \multicolumn{2}{|c|}{$\psi / \phi$} \\
\hline \multicolumn{8}{|c|}{ Shot $44669 \mathrm{~A}$} \\
\hline 0.30 & 3.58 & -50.9 & 0 & 0.268 & 2.18 & 1.07 & 0 \\
\hline 0.50 & 2.49 & -2.00 & 0.882 & 0.148 & 3.30 & -0.0746 & 0.0498 \\
\hline 0.50 & 2.49 & -33.9 & 0 & 0.148 & 3.30 & 1.09 & 0 \\
\hline 0.70 & 1.40 & -1.39 & 0.367 & 0.0699 & 5.14 & -0.105 & 0.0468 \\
\hline \multicolumn{8}{|c|}{ Shot $44669 \mathrm{~B}$} \\
\hline 0.30 & 3.11 & -3.93 & 2.55 & 0.267 & 2.57 & -0.0403 & 0.103 \\
\hline 0.30 & 3.11 & -39.8 & 0 & 0.267 & 2.57 & 1.12 & 0 \\
\hline 0.50 & 1.88 & -1.29 & 1.36 & 0.148 & 3.86 & -0.0259 & 0.0843 \\
\hline 0.50 & 1.88 & -22.9 & 0 & 0.148 & 3.86 & 1.11 & 0 \\
\hline 0.70 & 1.06 & -0.482 & 0.969 & 0.070 & 6.07 & 0.00414 & 0.117 \\
\hline
\end{tabular}
tic electrons. In the electrostatic limit with adiabatic electrons, the result gives the 44669 A maximum growth rate

TABLE V. Growth rates and real frequencies obtained from the dimensionless local electromagnetic dispersion relation at various radii for the discharge 44669. 


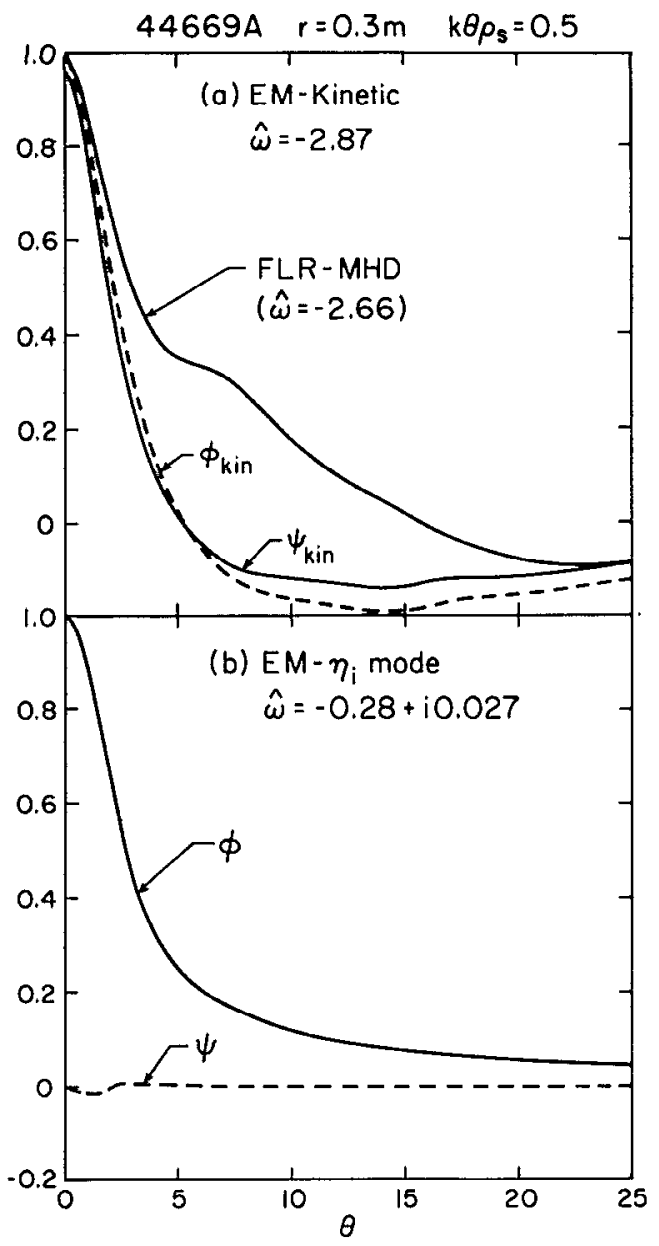

FIG. 6. The eigenfunctions of the electromagnetic kinetic and FLR-MHD ballooning modes. In (a), the stable kinetically modified FLR-MHD mode is given from Eq. (13) (kinetic) and Eq. (13) (FLR-MHD). In (b), the electrostaticlike mode from the electromagnetic equation (15) is given.

$\gamma_{m}=9 \times 10^{4} / \mathrm{sec}$ and the $44669 \mathrm{~B}$ value $\gamma_{m}=6.5 \times 10^{4} / \mathrm{sec}$ at $r=0.15 \mathrm{~m}$.

Within the adiabatic approximation for the electron dynamics, the electromagnetic corrections are weak. However, the electromagnctic effects appear to be substantial at the inner radii when the assumption of adiabatic electron dynamics is released. For example, the integral equation analysis shows that, at $r=0.15 \mathrm{~m}$, the electromagnetic-nonadiabatic electron systems have a growth rate of only $\gamma=4 \times 10^{3} / \mathrm{sec}$ for $44669 \mathrm{~A}$ and $\gamma=4.1 \times 10^{4} / \mathrm{sec}$ for 44669 $\mathrm{B}$, which are notably smaller than the values given above for electrostatic limit with adiabatic electrons.

Because of the stabilizing effect of finite beta, some favorable radial dependence of $\chi_{i}(r)$ can be produced but the effect is found to be weak for discharge 44669.

The quasilinear heat flux taking into account the inductive electric field $\partial A_{\|} / \partial t$ and the perturbed magnetic field $\delta B_{1}$ for both the ions and the electrons is given by

$$
\begin{aligned}
Q_{j}= & -\frac{c^{2} n_{j} T_{j}}{B^{2}} \sum_{\mathbf{k}} \int d^{3} \mathbf{v} d \omega\left[\frac{1}{T_{j}} \frac{d T_{j}}{d x}\left(\frac{m_{j} v^{2}}{2 T_{j}}-\frac{3}{2}\right)\right. \\
& \left.+\frac{1}{n_{j}} \frac{d n_{j}}{d x}-\frac{\omega e_{j} B}{c k_{y} T_{j}}\right]\left(\frac{m v^{2}}{2 T_{j}}\right) \pi \delta\left(\omega-k_{\|} v_{\|}\right)
\end{aligned}
$$

$$
\times k_{y}^{2} J_{0}^{2}\left(k_{\perp} \rho\right)\left|\phi_{\mathrm{k} \omega}(x)-\frac{v_{\|}}{c} A_{\| \mathbf{k} \omega}(x)\right|^{2}
$$

Because of the slablike resonance approximation, this $Q_{j}$ is proportional to $\left|E_{\| \mathbf{k} \omega}(x)\right|^{2} / k_{\|}^{2}(x)$. For $Q_{i}$ only, the large $k_{\|}$ part of the wave number spectrum contributes and, for $Q_{e}$, only the small $k_{\|}$part of the spectrum contributes significantly. The solution of Ampère's law for $A_{\|}$shows that $A_{\|}$is not negligible compared with $\phi$, so that the effective crossfield correlation length is increased by the finite beta coupling to $A_{\|}$. Using the electromagnetic quasilinear diffusion coefficients from Eq. (16) evaluated at the mixing length amplitude

$$
(\Delta x)^{2}=\left|\operatorname{Re} \frac{\int x^{2}\left[E_{\|}(x)\right]^{2} d x}{\int\left[E_{\|}(x)\right]^{2}}\right|,
$$

results in the electromagnetic diffusivity estimate of $\chi_{i}=\gamma(\Delta x)^{2}=0.77 \mathrm{~m}^{2} / \mathrm{sec}\left(k_{y} \rho_{s}=0.3\right)$ compared with the electrostatic value of $\chi_{i}=12 \mathrm{~m}^{2} / \mathrm{sec}\left(k_{y} \rho_{s}=0.5\right)$ for $44669 \mathrm{~A}$ at $r=0.15 \mathrm{~m}$. It is worth mentioning that $\chi_{i}=2.45$ $\mathrm{m}^{2} / \mathrm{sec}$ and $\chi_{i}=0.30 \mathrm{~m}^{2} / \mathrm{sec}$ are obtained, respectively, from electrostatic and electromagnetic perturbations if $\chi_{i}=\gamma / k_{y}^{2}$ is used to estimate the diffusivity. The differences are sufficient to indicate that, for the slablike modes, the electrostatic approximation is breaking down in the plasma core.

\section{OTHER STABILITY AND TRANSPORT EFFECTS}

\section{A. Effect of steeper edge gradients}

The ion temperature $T_{i}$ is only measured at a small set of discrete radial points, and it is conceivable that the $T_{i}(r)$ profile is not a simple smooth function but may have smallscale oscillations with some regions of large gradients $d T / d r$. In order to test the sensitivity of our theoretical $\chi$ 's to the experimentally measured gradients, "what if" numerical experiments are performed first of reducing $L_{T_{i}}$ and then reducing both $L_{T_{1}}$ and $L_{n}$ by one-half at the radius $r=0.7 \mathrm{~m}$. The results from local kinetic theory for $44669 \mathrm{~A}$ state are that the growth rates and $\chi_{i}$ increase to about two times the reference value for both the two gradient variations. Even these large changes in $L_{i_{i}}$ and $L_{n_{i}}$ seem to be insufficient to explain the experimental diffusivity result, which is over 10 $\mathrm{m}^{2} / \mathrm{sec}$ at $r=0.7 \mathrm{~m}$.

\section{B. Effect of carbon impurity}

The dominant impurity is fully ionized $(Z=6)$ carbon. Since the gradient scale lengths for carbon are not well known, we first studied the effect of including the carbon component $\beta_{c}=n_{c} / n_{e}$ in Eq. (1) within the slab approximation with $\omega_{*}(C)=0$. The growth rates are reduced to about one-half their $\beta_{c}=0$ value when $\beta_{c}=0.1$ (or $Z^{2} n_{c} / n_{e} \simeq 3.6$ ) in the A state and, in the B state, the effect is weaker with a reduction of about $2 / 3$ in the growth rate. Taking $\omega_{*}(C) \simeq-\omega_{* e}$ leads to stronger stabilization with 
the A state becoming stable for $\beta_{c} \gtrsim 0.05$ and the stability of the $B$ state is, again, less affected by the carbon component.

\section{Comparison with the Swedish stability and transport analysis}

The plasma theory group ${ }^{27}$ at Chalmers University, Sweden has developed a model of the stability and transport for the ion temperature and collisionless trapped electron tempcrature gradient instability that is reported to produce agreement with the power balance thermal diffusivities in some JET and TEXTOR discharges. ${ }^{28}$ Their theoretical model differs from that presented here in the following aspects. The model uses collisionless two-component hydrodynamic equations in which the toroidal drift frequency $\omega_{D}$ is taken as completely dominant over the $k_{\|} v_{i}$ resonance. The effect of magnetic shear is neglected as is the role of ion acoustic waves. The recent version ${ }^{29}$ of their theory contains a continued-fraction approximation for the reactive part of the drift resonance $\omega=\omega_{D}$, which seems to provide a fairly good fit to the $k_{\|}=0$ kinetic results, even though the dissipative part (due to collisionless phase mixing) is ignored. (Extension to include the dissipative part might be done with a variation of the work of Hammett and Perkins.) Their theoretical modeling assumes the modes to be localized to the outside $(\theta=0)$ of the torus and that the spectrum of $k_{k}$ is sufficiently small to be negligible compared with the toroidal drift $\omega_{D}$ effects. The key parameters are then $L_{n} / R$ and $L_{T_{i}} / R$, as well as the trapped electron fraction $f_{t}$ and $\tau=T_{e} / T_{i}$. In the Swedish model, the stability, determined by the roots of a fourth-order polynomial in $\omega$, gives a trapped electron mode rotating in the electron diamagnetic direction, which is used to calculate $\chi_{e}$ and an $\eta_{i}$ mode rotating in the ion direction, which is used to determine the $\chi_{i}$. We have solved their polynomial dispersion relation for TFTR discharge (44669) and find that their growth rates for the mode rotating in the ion direction are somewhat smaller than those obtained in the kinetic analysis in Sec. III A. A typical comparison is that, in the A state at $r=0.5$ $\mathrm{m}$ before injection, their equation gives the ion mode $\omega+i \gamma=-0.612+0.0537 i$ and the electron mode $\omega+i \gamma=0.224+0.106 i$ compared with the local kinetic value $\omega+i \gamma=-0.274+0.148 i$ in unit $c_{s} / L_{n}$, where the adiabatic electron model used in Eq. (2) gives only the ion mode.

The second major difference is in the formula used for the amplitude of the potential fluctuations by the Swedish group. They modify the mixing length level by including a factor of $\gamma / \omega_{*}$ so that the quasilinear formula for $\chi_{i}$ is now proportional to

$$
\gamma^{3} /\left[\left(\omega-\frac{5}{3} \omega_{D}\right)^{2}+\gamma^{2}\right]\left\langle k_{x}^{2}\right\rangle .
$$

This makes their $\chi$ formulas vanish at the rate $\gamma^{3}$ as $\gamma \rightarrow 0$ as it does from large compressibility when $L_{n} / R \geqslant 1$ in the plasma core. In this way, the resulting $\chi_{i}$ develops a radial profile that is closer in shape to the power balance $\chi_{i}(r)$ than that reported here in Table III. Nordman et al. ${ }^{27}$ support their choice of the modified mixing length formula by appealing to agreement with a simple two-dimensional toroidal mode coupling simulation, which has no magnetic shear or $\omega_{D i}(\theta)$ variation. Their results for $\chi_{i}$ would appear, however, to contradict both the theoretical and simulation results obtained by Hamaguchi and Horton, ${ }^{8}$ where the $\chi_{i}$ is shown to vary as $\eta_{i}-\eta_{i, \text { crit }}$ at small $\gamma$, which is no faster than $\gamma^{2}$ and is close to $\gamma$ for the slab model. Of course, the problem of obtaining accurate theoretical formulas for the saturation level is an unsolved problem, which leaves room for various models. For the near marginal states $\gamma \rightarrow 0$, bifurcation analysis ${ }^{8}$ gives a systematic calculation of the variation $\chi_{i}=\left(\eta_{i}-\eta_{i, \text { crit }}\right) \chi_{1}$, which does not agree with the $\gamma^{3}$ variation in Nordman et al. ${ }^{27}$ For comparison, we have applied the $\chi$ formulas of Nordman et $a l^{27}$ to the TFTR discharge. We find that their extra power of $\gamma / \omega_{*}$ in the fluctuation level formula and the $s=0$ approximation have the effect of making the $\chi_{i}(r)$ increase with radius. The formulas have the problem, however, of predicting that $\chi_{e} \geqslant 4 \chi_{i}$, contrary to theoretical expectations and to the power balance diffusivities that have ${ }^{3,4} \chi_{i}>\chi_{e}$. For $\gamma_{k}^{2}>\left(\omega_{k}-\frac{5}{3} \omega_{D}\right)^{2}$, the Nordman et $a l^{27} \chi_{i}$ formula reduces to the usual estimate of $\gamma_{k} / k^{2}$, consistent with Hamaguchi and Horton away from marginal stability.

Rewoldt and Tang $^{30}$ find a different behavior for the effect of the trapped electron mode. They find one eigenmode with a Gaussian-like $\phi_{k_{\theta}}(\theta)$ that changes direction of rotation from the ion diamagnetic to electron diamagnetic as $\eta_{i}$ is decreased below 1 to 1.5 . They call this continuous root the hybrid mode. When the mode rotates in the electron direction, the growth rate has an enhancement due to the trapped electron contribution. For larger $\eta_{i}$, the growth is determined by the ion dynamics with $\gamma \simeq 1.25\left(1+\eta_{i}\right) \times 10^{4}$ sec for $k_{\theta} \rho_{s}=0.356, r / a=0.21$ in the beam-heated TFTR discharge 22014. Their quasilinear transport studies show $\chi_{i}>\chi_{e}$ with $\chi_{i}$ and $\chi_{e}$ comparable to those obtained from power balance at the mixing length level.

One may conclude from these comparisons of theory with experiment, as is also obvious from the proportionality of the quasilinear thermal flux with the square of the amplitude, that the actual fluctuation levels increase more strongly toward the outside than given by the mixing length level formula as presently understood and applied. The problem of the disagreement in the radial profile of $\chi_{t}$ may be removed if actual measured fluctuation levels are used in the quasilinear formulas. A recent study by Bravenec et al. ${ }^{31}$ reports $\chi_{e}(r)$ using the measured fluctuation levels in the quasilinear formula in a study of electron power balance in TEXT. The study, however, still shows disagreement in the radial profile of $\chi_{e}(r)$ in the outer edge region of the Ohmic TEXT experiment. In all tokamaks, in both the $\mathrm{L}$ - and $\mathrm{H}$ confinement modes, the measured fluctuation levels are, to the authors' knowledge, strongly increasing toward the plasma edge. The problem with the radial profiles $\chi_{i}(r)$ and $\chi_{e}(r)$ then appears to reduce to the fact that the mixing length fluctuation levels $\Delta X / L_{T_{i}}$ and $\Delta X / L_{n}$, given by theoretical formulas used to obtain $\chi_{i}$, do not increase rapidly enough with radius. Perhaps, it is necessary to find more directly the mixing scales in the edge turbulence and to consider the long-correlated $\mathbf{E} \times \mathbf{B}$ drift orbits that occur in regions where the vortex rotation parameter ${ }^{9}$ $R_{E}=k_{1} \tilde{v}_{E} / \Delta \omega>1$. 


\section{Trapped electron destabilization}

When the profiles are such that the ion-temperaturegradient-driven turbulence is weak, it is necessary to calculate $\chi$, taking into the resonant trapped electron response as a drive to the same electrostatic drift modes $D_{\mathrm{ES}}(k, \omega)=0$. In classical $\eta_{i}$-mode theory, the resonant electron response is neglected since the contribution is subdominant for large $\eta_{i}$ and of a different physical origin.

Because of the fast electron transit $v_{e} / q R$ and bounce $\epsilon^{1 / 2} v_{e} / q R$ frequencies (with $\epsilon=r / R$ ) compared with the fluctuation frequencies, the electron response $\tilde{n}_{e}$ is a bounce average of $\phi(\theta)$ over the parallel electron motion $\dot{\theta}=v_{\|} / q R= \pm[2(E-\mu B)]^{1 / 2} / q R$. For $B \simeq B_{0}(1$ $-\epsilon \cos \theta)$ and the pitch angle variable $\lambda=\mu B_{0} / E$, the argument of the elliptic function integrals $K(m)$ and $E(m)$ is given by

$$
\begin{aligned}
m_{\lambda}=\kappa^{2} & =\frac{1}{2}\left(1+\frac{1-\lambda}{\epsilon}\right) \\
& = \begin{cases}0 & \text { at } \lambda=1+\epsilon \text { (deeply trapped) } \\
1 & \text { at } \lambda=1-\epsilon \text { (separatrix). }\end{cases}
\end{aligned}
$$

The pitch angle averaged trapped electron resonance is

$$
\begin{aligned}
H_{e}^{\operatorname{Tr}}(k, \omega, w)= & \int_{1-\epsilon}^{1+\epsilon} \frac{d \lambda}{\pi} \\
& \times \frac{\tau\left(m_{\lambda}\right)}{\omega-\epsilon_{n} \omega_{*} w G\left(m_{\lambda}, s\right)+i v_{\mathrm{eff}} / w^{3 / 2}},
\end{aligned}
$$

with the reduced quarter-bounce period $\tau\left(m_{\lambda}\right)=K\left(m_{\lambda}\right)$ $\times(2 / \lambda \epsilon)^{1 / 2}$, and

$G\left(m_{\lambda}, s\right)=\left(-1+\frac{2 E\left(m_{\lambda}\right)}{K\left(m_{\lambda}\right)}\right)+2 s\left(\frac{2 E\left(m_{\lambda}\right)}{K\left(m_{\lambda}\right)}-2+2 m_{\lambda}\right)$.

The trapped electron density response function is then

$$
\tilde{n}_{e}=\left(n_{e} e \Phi / T_{e}\right)\left(1-P_{e}^{\mathrm{Tr}}\right)
$$

with

$$
\begin{aligned}
P_{e}^{\mathrm{Tr}}= & \frac{2}{\pi^{1 / 2}} \int_{0}^{\infty} d w w^{1 / 2} e^{-w}\left\{\omega-k_{y}[1\right. \\
& \left.\left.+\eta_{e}\left(w-\frac{3}{2}\right)\right]\right\} H_{e}^{\mathrm{Tr}}(k, \omega, w) .
\end{aligned}
$$

The classical theory ${ }^{32}$ of the trapped electron mode follows from $D_{E S}^{T_{m}}=1-P_{e}^{T_{\mathrm{T}}}+\tau\left[1-P_{i}^{f}(\omega, k)\right]=0$, where the nonresonant or hydrodynamic ion response function $P_{i}^{H}(\omega, k)$ is taken for the ions and the resonant electron response $\operatorname{Im} P_{e}^{\mathrm{Tr}}$ drives the turbulence through $\gamma_{k}=\operatorname{Im} P_{e}^{\mathrm{Tr}} /\left(\partial D_{\mathrm{ES}} / \partial \omega\right)$. The nonadiabatic electron response in Eq. (19) gives a phase shift between $\tilde{n}_{e}$ and $\widetilde{\Phi}$ leading to particle transport.

For $44669 \mathrm{~A}$ at $r=0.3 \mathrm{~m}$ with $\eta_{e}=1.22$, we find two roots of the electrostatic dispersion relation with $P_{e}^{T_{\mathrm{r}}}$. The root with the largest $\gamma$ has the behavior shown in Fig. 1 of Rewoldt and Tang ${ }^{30}$ when $\eta$ is varied from -5 to +3 . For $\eta_{i} \gtrsim 2$ the growth rate is dominated by the $\eta_{i}$ driving mechanism. The second root has a considerably smaller growth rate with $\gamma_{2} / \gamma_{1} \leqslant 1 / 6$.
The trapped-electron driven turbulence produces $\mathbf{E} \times \mathbf{B}$ turbulent diffusion of the ions and electrons given in the quasilinear approximation by

$$
\begin{aligned}
\left(\begin{array}{c}
\Gamma \\
q_{e}
\end{array}\right)= & \frac{n_{e} c T_{e}}{e B} \sum_{\mathbf{k} \omega}\left|\frac{e \Phi_{\mathbf{k}}}{T_{e}}\right|^{2} \frac{2}{\pi^{1 / 2}} \int_{0}^{\infty} d w w^{1 / 2} e^{-w} \\
& \times\left(\begin{array}{c}
1 \\
w-3 / 2
\end{array}\right)\left\{\omega-k_{y}\left[1+\eta_{e}\left(w-\frac{3}{2}\right)\right]\right\} \\
& \times \operatorname{Im} G_{e}^{\operatorname{Tr}_{r}}(\mathbf{k}, \omega, w),
\end{aligned}
$$

with the thermal fluxes $Q_{i}$ and $Q_{e}$ given by

$$
Q_{i}=\frac{3}{2} T_{i} \Gamma_{i}+q_{i}
$$

and

$$
Q_{e}=\frac{3}{2} T_{e} \Gamma_{e}+q_{e},
$$

where the $q_{i}$ and $q_{e}$ are the conductive part of the thermal flux due to (the out-of-phase) $\widetilde{T}_{j}$ fluctuations. At the mixing length level of turbulence, where

$$
\left(\tilde{n}_{k} / \bar{n}_{e}\right)^{2} \simeq 1 /\left\langle k_{x}^{2} L_{n}^{2}\right\rangle \approx\left(\rho_{s}^{2} / L_{n}^{2}\right)\left(L_{s} / L_{n}\right),
$$

these fluxes from the trapped electrons can explain the magnitude and some of the parametric variations found in tokamaks. ${ }^{31,33,34}$ In Bravenec et al., ${ }^{31}$ the fluctuation spectrum measured by FIR scattering and the heavy ion-beam probe are used in the quasilinear formulas (21)-(23). The principal difficulty with using the trapped electron mode for $\chi_{e}$ in all regimes is the lack of a sufficiently strong $q$ dependence and the tendency for the $q_{e}(r)$ flux to decrease rapidly with increasing radius $r / a$ just as is the problem discussed above for the $\eta_{i}$-driven $\chi_{i}$ formulas.

A second source of electron thermal flux is obtained by including the short-wavelength $\nabla T_{e}$-driven electromagnetic turbulence. This small-scale $\eta_{e}$-driven turbulence, which is the electron analog of the $\eta_{i}$, produces a collisionless skin depth electromagnetic $\chi_{e}$ given by trapped electrons

$$
\chi_{e}=\epsilon^{1 / 2} \omega_{b e} c^{2} / \omega_{p e}^{2}=r v_{e} c^{2} / q R^{2} \omega_{p e}^{2} .
$$

As shown in Table II, rate $\chi_{e}^{(1)}$ from Eq. (24) can exceed that from the longer-wavelength part of the spectrum $\chi_{e}^{(2)}$. For fixed $T_{e}$, the electromagnetic $\chi_{e}$ in Eq. (24) vanishes with electron mass $m_{e} \rightarrow 0$ as $m_{e}^{1 / 2}$, whereas the electrostatic $\chi_{e}$ in Eq. (21) is independent of $m_{e}$, which indicates the physically different origins of these transport components. The relationship between the $\chi_{e}^{(1)}$ skin depth transport and the $\chi_{e}^{(2)}$ trapped electron mode is analyzed in $\mathrm{Kim}$ et al ${ }^{35}$

\section{CONCLUSION}

The TFTR supershot density-modification experiment of Zarnstorff $e t a l^{5,6}$ has been analyzed for local and ballooning mode stability to the $\eta_{i}$ modes. The analysis shows that, even though the ion temperature gradient parameter $\eta_{i}$ increases almost an order of magnitude $\left(\eta_{i}=2.3 \rightarrow 21\right.$ at $r=0.3 \mathrm{~m}$ ) from the flattening of the density profile, the growth rate and wave number of the dominant $\eta_{i}$ modes are not strongly changed. The ion thermal diffusivity constructed from the linear kinetic growth rate and the isotropic turbulent correlation length $\Delta X_{\mathrm{ml}} \sim k_{m}^{-1}$ as required by the 3$\mathrm{D}$ turbulence simulations, yields a decrease in $\chi_{i}$ in the post 
injection state due to ( 1 ) the lowering of the ion and electron temperatures and (2) the increase of the toroidicity parameter $\epsilon_{n}$ to the order of unity where compressibility is strongly stabilizing. The toroidal $\eta_{i}$-mode growth rate $\gamma_{k}\left(\epsilon_{n}, \eta_{i}\right)$ has a maximum at small $\epsilon_{n}$ for fixed $\eta_{i}$.

The stability analysis from both the local and nonlocal equations shows that the discharges are not near marginal stability. Even within the classical $\eta_{i}$-mode approximation of adiabatic electrons, the $\nabla T_{i}$-driven modes are unstable both before and after pellet injection. The ion thermal diffusivities derived here are not sufficiently large to force the profiles to marginal stability.

The previous theories predicted $\chi_{i}$ 's that were much larger than observed in the experiment. Our present calculations do a better job by including a number of important effects that were not adequately treated in previous theories. Finite-gyroradius and kinetic effects (such as Landau damping) are retained, which reduce the growth rate significantly, which is partially of set by the inclusion of toroidal driving terms that had been missing from many of the previous theories that were in slab geometry. Also, we use a shorter mixing length $1 /\left|k_{y}\right|$ rather than a longer mixing length, which sometimes is suggested by the linear radial mode structure but which is not expected to survive the nonlinear regime.

There are a number of ways in which future work could build upon our calculations. We have employed local toroidal calculations that are good for arbitrary $k_{\|} v_{t i} / \omega$ but which assume a $k_{\|}=1 /(q R)$, and we have used a ballooning mode equation expanded for small $k_{\|} v_{t i} / \omega$ but which self-consistently determines a linear spectrum of $k_{\|}$'s. Although we have found good agreement between the two approaches, it would be interesting to repeat the stability analysis using more complicated ballooning codes that do not assume small $k_{\|} v_{t i} / \omega$. We have used a simple $s$ - $\alpha$ model equilibrium, but a more accurate equilibrium would, among other things, introduce a nonlocal dependence on integral quantities through the Shafranov shift $\Delta(r)$. At the edge of this plasma $(r \geqslant 0.65 \mathrm{~m}), \quad \Delta^{\prime}=-(a / R)\left(\beta_{p}+l_{i} / 2\right)$ $=-0.65$ is large, giving an enhancement in the pressure gradient by a factor of 2 to 3 while also shortening the connection length by a similar factor.

The present $\eta_{i}$ mode theories fail, however, to explain the radial dependence of the power balance $\chi_{i}$ over all radii, in particular, at the outer edge region. Attempts to obtain the observed increase of $\chi_{i}(r)$ with $r / a$ by adding the effects of (i) the finite-beta drift wave-MHD mode coupling, (ii) the slablike mode, or (iii) the trapped electron resonances are found to be inadequate. The tracking $0.2 \leqslant \chi_{e} / \chi_{i} \leqslant 0.7$ suggests that both the $\nabla T_{i}$ and the collisionless-trapped-electron driving mechanisms are operating. The disagreement in the $\chi_{i}$ and $\chi_{e}$ profiles appear to arise from the underestimate by theory of the actual fiuctuation levels that are measured to increase strongly with radius.

The problem of the lack of agreement in the radial variation of the theoretical turbulent conductivities compared with the experimental power balance conductivities occurs for many forms of microturbulence. The conflict suggests one of several possibilities: (i) that another class of linear instabilities is controlling transport in the outer regions, (ii) that either the relevant gradient scale lengths $L_{n}(r), L_{T}(r)$ in the dynamics of the plasma are substantially shorter in the region $r / a>0.5$ than reported from the measured mean (temporally and spatially smoothed) profiles $n_{j}(r), T_{j}(r)$, or (iii) that the actual mixing length $\Delta X_{\mathrm{ml}}$ is substantially greater than the theoretical values. Indeed, there is evidence on a number of tokamaks that the theories underestimate the actual fluctuation levels that are measured to increase strongly with radius. Theoretical transport effects outside the scope of the usual locally homogeneous turbulence models related to the radial profiles of the gradient parameters, the shear profile, and the $\mathbf{E} \times \mathbf{B}$ shear flows created in the outer layer of the plasma may be responsible for the larger edge transport. The effect of a strongly localized $\mathbf{E} \times \mathbf{B}$ shear flow layer ${ }^{36-38}$ can distort the radial wave functions, and thus increase the radial mixing length. Studies of the shear flow layer effect are advancing and show that, if the shear flow scale length $L_{E}$ for the nonuniform flow $v_{E}(r)$ satisfies $v_{E}^{\prime}=v_{E} / L_{E}>\left(c_{s} / L_{s}\right)$, the Kelvin-Helmholtz-like vortices are formed between the counter-flowing plasma streams. In this shear flow layer, the mixing width $\Delta X_{\mathrm{ml}}$ becomes as large as the vortex diameter, which is found to approach $L_{E}$ for sufficiently small $L_{E}{ }^{38}$

Other mechanisms for increasing the mixing length may be the electromagnetic shielding of the induced parallel current filaments by the collisionless skin depth $c / \omega_{p e}$, which exceeds $\rho_{s}\left(L_{s} / L_{n}\right)^{1 / 2}$ at sufficiently low $\beta_{e}$ characteristic of the edge region, ${ }^{9,35}$ and long-correlated $\mathbf{E} \times \mathbf{B}$ orbits in the amplitude regime above the mixing length level. ${ }^{39}$

In this work, we have clarified the basic problems that occur in using present theoretical models for understanding and analyzing power balance in the supershot regime in the TFTR.

\section{ACKNOWLEDGMENTS}

The authors thank Professor D. E. Baldwin for his encouragement and critical comments on the project during his tenure as Director of the Institute for Fusion Studies. We thank P. C. Efthimion, G. Schmidt, and the rest of the TFTR team at the Princeton Plasma Physics Laboratory (PPPL) who (along with M. C. Zarnstorff) performed the original experiments and provided us with access to the data.

The work was supported by U.S. Department of Energy Contract No. DE-FG05-80ET-53088 and PPPL Contract No. DE-AC02-76-CHO-3073.

\section{APPENDIX: ELECTROMAGNETIC BALLOONING MODE EQUATIONS}

The calculations of the Vlasov parallel current and charge densities are given in Cheng, ${ }^{23}$ Hong et al., ${ }^{21}$ and Horton et al. ${ }^{40}$ The ballooning mode representation of the fields in a torus is used, and the mode frequencies are taken to be between the transit frequencies of the ions and electrons which is justified a posteriori. The condition of quasineutrality is 


$$
\hat{a} \phi+\hat{b} \psi=0
$$

and the parallel component of Ampère's law is

$$
\hat{b} \phi+\hat{d} \psi=0,
$$

where

$$
\begin{aligned}
& \hat{a}(k, \omega, \theta)=-1+\tau(P-1)-\frac{c_{s}^{2}}{\omega^{2} q^{2} R^{2}} \frac{\partial}{\partial \theta} P_{3} \frac{\partial}{\partial \theta}, \\
& \hat{b}(k, \omega, \theta)=1-\frac{\omega_{*_{e}}}{\omega}+\frac{c_{s}^{2}}{\omega^{2} q^{2} R^{2}} \frac{\partial}{\partial \theta} P_{2} \frac{\partial}{\partial \theta},
\end{aligned}
$$

and

$$
\begin{aligned}
\hat{d}(k, \omega, \theta)= & \frac{\rho^{2} v_{A}^{2}}{\omega^{2} q^{2} R^{2}} \frac{\partial}{\partial \theta} \nabla_{1}^{2} \frac{\partial}{\partial \theta}-\left(1-\frac{\omega_{*_{e}}}{\omega}\right) \\
& +\left(1-\frac{\omega_{*_{p e}}}{\omega}\right) \frac{\omega_{D_{e}}(\theta)}{\omega}-\frac{c_{s}^{2}}{\omega^{2} q^{2} R^{2} \partial \theta} \Gamma_{1} \frac{\partial}{\partial \theta} .
\end{aligned}
$$

The ion kinetic response functions, $P$ and $P_{j}(j=1,2,3)$, are given by

$$
\begin{aligned}
& P=\int d \mathbf{v} F_{i}(\mathrm{v})\left(\frac{\omega-\omega_{*_{i}}^{T}}{\omega-\omega_{D t}}\right) J_{0}^{2}\left(\frac{k_{\perp} v_{1}}{\omega_{c i}}\right), \\
& P_{j}=\int d \mathrm{v} F_{i}(\mathrm{v}) \frac{\omega^{j-1}\left(\omega-\omega_{*_{i}}^{T}\right)}{\left(\omega-\omega_{D t}\right)^{j}} \frac{m_{i} v_{\|}^{2}}{T_{i}} J_{0}^{2}\left(\frac{k_{\perp} v_{1}}{\omega_{c t}}\right),
\end{aligned}
$$

where $\quad F_{i}(\mathbf{v})=\left(2 \pi v_{i}^{2}\right)^{-3 / 2} \exp \left(-v^{2} / 2 v_{i}^{2}\right) \quad$ with $\quad v_{i}$ $=\left(T_{i} / m_{i}\right)^{1 / 2}$ and $\omega_{*_{i}}^{T}=\omega_{*_{i}}\left[1+\eta_{i}\left(v^{2} / v_{i}^{2}-3 / 2\right)\right]$. The fluid limit of the $P_{j}(j=1,2,3)$ functions is

$$
\begin{aligned}
P_{1}^{f}=P_{2}^{f}=P_{3}^{f} \cong & \left(1-\omega_{*_{i}} / \omega\right) \Gamma_{0}(b)-\left(\omega_{*_{i}} / \omega\right) \eta_{i}\left\{\Gamma_{0}(b)\right. \\
& \left.+b\left[\Gamma_{1}(b)-\Gamma_{0}(b)\right]\right\},
\end{aligned}
$$

with $\Gamma_{j}(b)=I_{j}(b) e^{-b}$ and $b=k_{1}^{2} \rho_{i}^{2}=k^{2} \rho_{i}^{2}\left(1+s^{2} \theta^{2}\right)$.

All frequencies are measured in units of $c_{s} / r_{n}$ and the wave number $k_{\theta}$ in units of $\rho_{s}=c\left(m_{i} T_{e}\right)^{1 / 2} / e B$. The dimensionless complex frequency $\omega\left[c_{s} / r_{n}\right]$ is a function of the seven dimensionless parameters $k, \beta_{e}, q, \epsilon_{n}, s, \eta_{i}$, and $\tau=T_{\mathrm{c}} / T_{i}$. With the dimensionless variables, we write Eqs. (A1) and (A2) as

$$
\begin{aligned}
& \left(1-\tau(P-1)+P_{3}^{f} \frac{\epsilon_{n}^{2}}{q^{2} \omega^{2}} \frac{\partial^{2}}{\partial \theta^{2}}\right) \phi \\
& =\left(1-\frac{\omega_{*_{e}}}{\omega}+P_{2}^{f} \frac{\epsilon_{n}^{2}}{q^{2} \omega^{2}} \frac{\partial^{2}}{\partial \theta^{2}}\right) \psi \\
& {\left[\frac{\epsilon_{n}^{2}}{q^{2} \omega^{2}} \frac{2}{\beta_{e}} \frac{\partial}{\partial \theta} k_{1}^{2} \frac{\partial}{\partial \theta}+\left(1-\frac{\omega_{*_{e}}}{\omega}\right)\left(1-\frac{\omega_{D_{e}}}{\omega}\right)\right.} \\
& \left.\quad+P_{1}^{f} \frac{\epsilon_{n}^{2}}{q^{2} \omega^{2}} \frac{\partial^{2}}{\partial \theta^{2}}\right] \psi=\left(1-\frac{\omega_{*_{e}}}{\omega}+P_{2}^{f} \frac{\epsilon_{n}^{2}}{q^{2} \omega^{2}} \frac{\partial^{2}}{\partial \theta^{2}}\right) \phi
\end{aligned}
$$

\section{Limiting regimes of the kinetic eigenmode equation}

First we consider the limit that allows ion acoustic coupling terms to be zero with $\omega_{A}=\left(\epsilon_{n} / q\right)\left(2 / \beta_{e}\right)^{1 / 2}$ fixed. If $q \rightarrow \infty$ but $q^{2} \beta_{\text {e }}$ finite, Eqs. (A6) and (A7) reduce to the second-order differential equation

$$
\begin{gathered}
{[1-\tau(P-1)]\left[\frac{\omega_{A}^{2}}{\omega^{2}} \frac{\partial}{\partial \theta^{2}} k_{1}^{2} \frac{\partial}{\partial \theta}+\left(1-\frac{\omega_{*_{e}}}{\omega}\right)\right.} \\
\left.\times\left(1-\frac{\omega_{D_{e}}}{\omega}\right)\right] \psi-\left(1-\frac{\omega_{*_{e}}}{\omega}\right)^{2} \psi=0
\end{gathered}
$$

where we used

$$
\frac{\psi}{\phi}=\frac{1+\tau(1-P)}{1-\omega_{*} / \omega}
$$

from Eq. (A6) and note that

$$
E_{\|}=i k_{\|} \phi(1-\psi / \phi) \text {. }
$$

The eigenmodes of Eq. (A8) have been analyzed in earlier works. ${ }^{21,23-25}$ With the full ion kinetic velocity space integral $P$, we give the results of the kinetic effects on MHD ballooning mode.

Equation (A8) also governs the toroidal $\eta_{i}$ mode in the low-beta limit

$$
\omega_{A}^{2} \rightarrow \infty \text { then } D_{\mathrm{ES}}(\omega, k)=1+\tau(1-P)=0,
$$

with the mode characteristics $(|\phi| \gg|\psi|)$. In the high-beta limit, Eq. (A8) reduces to the MHD ballooning mode $(|\phi| \sim|\psi|)$ for $k \rightarrow 0$ at finite $\omega$.

In the $\beta \rightarrow 0$ limit, Eq. (A8) reduces to

$$
[1-\tau(P-1)] \frac{\omega_{A}^{2}}{\omega^{2}} \frac{\partial}{\partial \theta} k_{1}^{2} \frac{\partial}{\partial \theta} \psi=0 .
$$

If we assume $D_{\mathrm{ES}}=[1-\tau(P-1)] \neq 0$, then we obtain the solution of Eq. (A11) as $\psi \sim \tan ^{-1} \theta$, which is an unphysical solution having $\int d \theta \psi^{2} \rightarrow \infty$. Thus, to have a solution that tends to zero for large $\theta$, we must have

$$
D_{\mathrm{ES}}(k, \omega, \theta=0)=[1-\tau(P-1)]=0,
$$

which is the local dispersion relation of toroidal $\eta_{i}$ mode.

Dispersion relation Eq. (A12) gives unstable $\eta_{i}$ mode when

$$
\eta_{i}>\eta_{c} \sim \frac{2}{3} \text { and } \epsilon_{T_{i}}=r_{T_{i}} / R<0.35 \text {. }
$$

Above the threshold, the mode has $\omega_{k} \simeq \omega_{D i}=-2 k \epsilon_{n}$ and $\gamma_{k} \sim v_{i} /\left(R r_{T_{i}}\right)^{1 / 2}$. Recent H-mode discharge experiments show inverted gradient profiles with $\eta_{i}<0$ and $\epsilon_{n}<0$. For the dissipative drift wave and the trapped electron mode, the inverted profiles show substantial gain in stability for $v_{*_{e}}<0.3$ regime. For a fixed or local value of $\theta$, the condition $\operatorname{Im} P=0$ yields the marginal stability frequency

$$
\omega_{m} \cong \frac{1+\frac{3}{2}\left|\eta_{i}\right|}{1-\left|\eta_{i}\right| /\left|\epsilon_{n}\right|} \omega_{*_{i}}
$$

and

$$
\operatorname{Re} P\left(\omega_{m}\right) \cong\left|\eta_{i} / \epsilon_{n}\right| \Gamma_{0}(b),
$$

which leads to the instability condition of

$$
\epsilon_{T_{i}}<\frac{\tau \Gamma_{0}(b)}{1+\tau}=\frac{\Gamma_{0}(b)}{1+T_{i} / T_{e}} \leqslant \frac{1}{1+T_{i} / T_{e}}
$$

for $\epsilon_{T_{i}}=r_{T_{i}} / R=\epsilon_{n} / \eta_{i}$ from a Nyquist diagram.

\section{Electrostatic toroidal integral equation}

In the limit where magnetic shear determines the mode structure and the drift velocities are taken local in $\theta$, the 
velocity integrals can be done without the expansion in $P_{\|} \sim k_{\|}^{2} v_{i}^{2} / \omega^{2}$ defined in Eq. (12). We obtain the integral equation

$$
(1+\tau) \phi\left(k_{x}\right)=\int_{-\infty}^{+\infty} K\left(k_{x}, k_{x}^{\prime}\right) \phi\left(k_{x}^{\prime}\right) d k_{x}^{\prime},
$$

where

$$
K\left(k_{x}, k_{x}^{\prime}\right)=\frac{2}{\sqrt{2 \pi}} \int_{-\infty}^{0} \frac{e^{-i \omega t} e^{-\left(k_{x}^{\prime}-k_{x}\right)^{2} / 4 \sigma_{t} t^{2}}}{\left(1+a_{t}\right) \sqrt{a_{t}}} G(t) d t,
$$

with the kinetic response function

$$
\begin{aligned}
G(T)= & \frac{\omega_{*_{e}}}{t \sqrt{2 \sigma_{t}}}\left[\frac{\omega}{\omega_{*_{e}}} \tau+1-\frac{3}{2} \eta_{i}+\frac{2 \eta_{i}}{\left(1+a_{t}\right)}\right. \\
& \times\left(1-\frac{k_{\perp}^{2}+k_{\perp}^{\prime 2}}{2 \tau\left(1+a_{t}\right)}+\frac{k_{\perp} k_{1}^{\prime}}{\tau\left(1+a_{t}\right)} \frac{I_{1}}{I_{0}}\right) \\
& \left.+\frac{\eta_{i}\left(k_{x}^{\prime}-k_{x}\right)^{2}}{4 a_{t} \sigma_{t} t^{2}}\right] \Gamma_{0}\left(k_{1}, k_{\perp}^{\prime}\right),
\end{aligned}
$$

where

$$
\begin{aligned}
& a_{t}=1+i\left(2 \epsilon_{n} \omega_{*_{e}} t / \tau\right), \\
& \sigma_{t}=\left(1 / \tau a_{t}\right)\left(L_{n} / L_{s}\right)^{2}, \\
& \Gamma_{0}\left(k_{1}, k_{1}^{\prime}\right)=I_{0}\left[k_{\perp} k_{1}^{\prime} / \tau\left(1+a_{t}\right)\right] \\
& \quad \times \exp \left[-k_{1}^{2}+k_{\perp}^{\prime 2} / 2 \tau\left(1+a_{t}\right)\right], \\
& k_{1}^{2}=k_{x}^{2}+k_{y}^{2}, \quad k_{1}^{\prime 2}=k_{x}^{\prime 2}+k_{y}^{2},
\end{aligned}
$$

and $\tau=T_{e} / T_{i}$. For $s \rightarrow 0$, the matrix $K\left(k_{x}, k_{x}^{\prime}\right)$ is diagonal and the eigenmodes are $\phi\left(k_{x}\right)=\delta\left(k_{x}-q\right)$ with the $q=0$ mode from Eq. (A15) giving the local toroidal dispcrsion in Eq. (2). For $|\omega| \gg k_{i l} v_{i}, \omega_{D i}$, the small $t$ limit $G(t)=G(0)$ $+G^{\prime}(0) t+\cdots$ of Eq. (A16) returns the differential equation valid for $P_{\|}<1$.

'S. M. Wolfe and M. Greenwald, Nucl. Fusion 26, 329 (1986).

${ }^{2}$ F. X. Söldner, E. R. Müller, F. Wagner, H. S. Bosch, A. Eberhagen, H. U. Fahrbach, G. Fussmann, O. Gehre, K. Gentle, J. Gernhardt, O. Gruber, W. Herrmann, G. Janeschitz, M. Kornherr, K. Krieger, H. M. Mayer, K. McCormick, H. D. Murmann, J. Neuhauser, R. Nolte, W. Poschenrieder, H. Röhr, K.-H. Steuer, U. Stroth, N. Tsois, and H. Verbeek, Phys. Rev. Lett. 61, 1105 (1988).

${ }^{3}$ S. D. Scott, P. H. Diamond, R. J. Fonck, R. J. Goldston, R. B. Howell, K. P. Jaehnig, G. Schilling, E. J. Synakowski, M. C. Zarnstorff, C. E. Bush, E. Fredrickson, K. W. Hill, A. C. Janos, D. K. Mansfield, D. K. Owens, H. Park, G. Pautasso, A. T. Ramsey, J. Schivell, G. D. Tait, W. M. Tang, and G. Taylor, Phys. Rev. Lett. 64, 531 (1990).

'S. D. Scott, V. Arunasalam, C. W. Barnes, M. G. Bell, M. Bitter, R. Boivin, N. L. Bretz, R. Budny, C. E. Bush, A. Cavallo, T. K. Chu, S. A. Cohen, P. Colestock, S. L. Davis, D. L. Dimock, H. F. Dylla, P. C. Efthimion, A. B. Erhrardt, R. J. Fonck, E. Fredrickson, H. P. Furth, R. J. Goldston, G. Greene, B. Grek, L. R. Grisham, G. Hammett, R. J. Hawryluk, H. W. Hendel, K. W. Hill, E. Hinnov, D. J. Hoffman, J. Hosea, R. B. Howell, H. Hsuan, R. A. Hilse, K. P. Jaehnig, A. C. Janos, D. Jassby, F. Jobes, D. W. Johnson, L. C. Johnson, R. Kaita, C. Kieras-Phillips, S. J. Kilpatrick, P. H. LaMarche, B. LeBlanc, R. Little, D. M. Manos, D. K. Mansfield, E. Mazzucato, M. P. McCarthy, D. C. McCune, K. McGuire, D. H. McNeill, D. M. Meade, S. S. Medley, D. R. Mikkelsen, R. Motley, D. Mueller, J. A. Murphy, Y. Nagayama, R. Nazakian, D. K. Owens, H. Park, A. T. Ramsey, M. H. Redi, A. L. Roquemore, P. H. Rutherford, G. Schilling, J. Schivell, G. L. Schmidt, J. Stevens, B. C. Stratton, W. Stodiek, E. J. Synakowski, W. M. Tang, G. Taylor, J. R. Timberlake, H. H. Towner, M. Ulrickson, S. von Goeler, R. Wieland, M. Williams, J. R. Wilson, K.-L. Wong, S. Yoshikawa, K. M. Young, M. C. Zarnstorff, and
S. J. Zweben, Phys. Fluids B 2, 1300 (1990).

${ }^{5}$ M. C. Zarnstorf, C. W. Barnes, P. C. Efthimion, G. W. Hammett, W. Horton, R. A. Hulse, D. K. Mansfield, E. S. Marmar, K. McGuire, G. Rewoldt, B. C. Stratton, E. J. Synakowski, W. Tang, J. Terry, X. Q. Xu, M. G. Bell, M. Bitter, N. L. Bretz, R. Budny, C. E. Bush, G. J. Fonck, E. D. Fredrickson, H. P. Furth, R. J. Goldston, B. Grek, R. J. Hawryluk, K. W. Hill, H. Hsuan, D. W. Johnson, D. C. McCune, D. M. Meade, D. Mueller, D. K. Owens, H. K. Park, A. T. Ramsey, M. N. Rosenbluth, J. Schivell, G. L. Schmidt, S. D. Scott, G. Taylor, and R. M. Wieland, in Plasma Physics and Controlled Nuclear Fusion Research, 1990, Proceedings of the 12th International Conference, Washington (IAEA, Vienna, 1991), Vol. I, p. 109.

${ }^{6}$ M. C. Zarnstorf, N. L. Bretz, P. C. Efthimian, G. Hammett, W. Horton, R. Hulse, D. Mansfield, E. Marmar, K. McGuire, G. Rewoldt, B. Stratton, E. Synakowski, W. Tang, J. Terry, X. Xu, M. Bell, M. Bitter, N. Bretz, R. Budny, C. Bush, R. Fonck, E. Fredrickson, H. Furth, R. Goldston, B. Grek, R. Hawryluk, K. Hill, H. Hsuan, R. A. Hulse, D. Johnson, M. McCune, D. Meade, D. Mueller, D. Owens, H. Park, A. Ramsey, M. Rosenbluth, J. Schivell, G. Schmidt, S. Scott, G. Taylor, and R. Wieland, in Proceedings of the 17th European Physical Society Conference on Controlled Fusion and Plasma Heating. Amsterdam (European Physical Society, Budapest, 1990), Vol. I, p. 39.

${ }^{7}$ H. Biglari, P. H. Diamond, and M. N. Rosenbluth, Phys. Fluids B 1, 109 (1989).

${ }^{8}$ S. Hamaguchi and W. Horton, Phys. Fluids B 2, 1833, 3040 (1990).

${ }^{9}$ W. Horton, Phys. Rep. 192, 177 (1990).

${ }^{10}$ G. W. Hammett and F. W. Perkins, Phys. Rev. Lett. 64, 3019 (1990).

${ }^{11}$ S. Hamaguchi and W. Horton, Plasma Phys. Controlled Fusion 34, 203 (1992).

${ }^{12}$ M. Kotschenreuther, H. L. Berk, R. Denton, S. Hamaguchi, W. Horton, C.-B. Kim, M. Lebrun, P. Lyster, S. Mahajan, W. H. Miner, P. J. Morrison, D. W. Ross, R. D. Sydora, T. Tajima, J. B. Taylor, P. M. Valanju, H. V. Wong, S. Y. Xiao, and Y. Z. Yang, in Plasma Physics and Controlled Nuclear Fusion Research, 1990. Proceedings of the 13th International Conference, Washington (IAEA, Vienna, 1991), Vol. II, p. 361.

${ }^{13}$ J. Y. Kim and W. Horton, Phys. Fluids B 3, 1167 (1991).

${ }^{14}$ R. R. Dominguez and R. E. Waltz, Phys. Fluids 31, 3147 (1988).

${ }^{15}$ W. Horton, B. G. Hong, and W. M. Tang, Phys. Fluids 31, 2971 (1988). ${ }^{16}$ F. Romanelli, Phys. Fluids B 1, 1018 (1989).

${ }^{17}$ T. S. Hahm and W. M. Tang, Phys. Fluids B 1, 1185 (1989)

${ }^{18}$ X. Q. Xu and M. N. Rosenbluth, Phys. Fluids B 3, 627 (1991).

${ }^{19}$ S. C. Cowley, R. M. Kulsrud, and R. V. Sudan, Phys. Fluids B 3, 2767 (1991).

${ }^{20}$ R. R. Dominguez and M. N. Rosenbluth, Nucl. Fusion 29, 844 (1989).

${ }^{21}$ B. G. Hong, W. Horton, and D. I. Choi, Plasma Phys. Controlled Fusion 31, 1291 (1989); B. G. Hong, W. Horton, and D.-I. Choi, Phys. Fluids B 1,1589 (1989).

${ }^{22}$ W. Horton, D. I. Choi, and W. M. Tang, Phys. Fluids 24, 1077 (1981).

${ }^{23}$ C. Z. Cheng, Nucl. Fusion 22, 773 (1982).

${ }^{24}$ W. M. Tang, G. Rewoldt, C. Z. Cheng, and M. S. Chance, Nucl. Fusion 25, 151 (1985).

${ }^{25}$ R. R. Dominguez and R. W. Moore, Nucl. Fusion 26, 85 (1986).

${ }^{26}$ J. Q. Dong, P. N. Guzdar, and Y. C. Lee, Phys. Fluids 30, 2694 (1987).

${ }^{27}$ H. Nordman, J. Weiland, and A. Jarmen, Nucl. Fusion 30, 983 (1990).

${ }^{28}$ A. Rogister, G. Hasselberg, F. Waelbroeck, and J. Weiland, Nucl. Fusion 28,1053 (1988).

${ }^{29}$ J. Nilsson, M. Liljestrom, and J. Weiland, Phys. Fluids B 2, 2568 (1990),

${ }^{30} \mathrm{G}$. Rewoldt and W. M. Tang, Phys. Fluids B 2, 318 (1990); Bull. Am. Phys. Soc. 35, 1981 (1990).

${ }^{31}$ R. Bravenec, D. W. Ross, P. M. Schoch, D. I. Brower, J. W. Heard, R. L. Hickok, P. W. Terry, A. J. Wootton, and X. Z. Yang, Nucl. Fusion 31, 687 (1991).

${ }^{32}$ M. Tang, Nucl. Fusion 18, 1089 (1978).

${ }^{33}$ W. Horton and R. D. Estes, Nucl. Fusion 19, 203 (1979).

${ }^{34}$ R. E. Waltz, Phys. Fluids 26, 169 (1983).

${ }^{35}$ D. E. Kim, D.-I. Choi, W. Horton, F. N. Yushmanov, and V. V. Parail, Phys. Fluids B 2, 547 (1990).

${ }^{36}$ S. Hamaguchi and W. Horton, Phys. Fluids B 4, 319 (1992).

${ }^{37}$ Y. B. Kim and P. H. Diamond, submitted to Phys. Fluids B.

${ }^{38}$ T. Tajima, W. Horton, P. J. Morrison, J. Schutkeker, T. Kamimura, K. Mima, and Y. Abe, Phys. Fluids B 3, 839 (1991).

${ }^{39}$ M. B. Isichenko and W. Horton, Comments Plasma Phys. Controlled Fusion 14, 249 (1991)

${ }^{40}$ W. Horton, J. E. Sedlak, D. I. Choi, and B. G. Hong, Phys. Fluids 28, $3050(1985)$. 